\title{
Catalytic Asymmetric Synthesis of Quaternary Carbons Bearing Two Aryl Substituents. Enantioselective Synthesis of 3-Alkyl-3-Aryl Oxindoles by Cataytic Asymmetric Intramolecular Heck Reactions
}

\author{
Amy B. Dounay, Keiko Hatanaka, Jeremy J. Kodanko, Martin Oestreich, Larry E. Overman*, \\ Lance A. Pfeifer and Matthew M. Weiss \\ Department of Chemistry, 516 Rowland Hall, University of California, Irvine CA 92697-2025
}

\section{Supporting Information}

Experimental procedures for the preparation of 11a-k, 18a-j, hydroxyl ethyl derivatives $\mathbf{1 8} \mathbf{a}^{\prime}, \mathbf{1 8} \mathbf{b}^{\prime}, \mathbf{1 8 d}$ ', 18g', 20-25, 26a, 27a, 27b, 28a, 28b, 30; copies of ${ }^{1} \mathrm{H}$ and ${ }^{13} \mathrm{C}$ NMR spectra for $11 \mathbf{a}-\mathbf{k}, \mathbf{1 8 a}-\mathbf{j}, \mathbf{1 8 a}$, 18b', 18d', 18g', 20-25, 26a, 27a, 27b, 28a, 28b, 30; HPLC traces used to determine the enantiopurity of 18a', 18b', 18c, 18d', 18e, 18f, 18g', 18h, 18i, 18j, 23, 24, and 30; graphical data for the thermal equilibration of atropisomers 23 and 24.

\section{Part 2}


Supporting Information January 28, 2003 Dounay, Hatanaka, Kodanko, Oestreich, Overman*, Pfeifer and Weiss

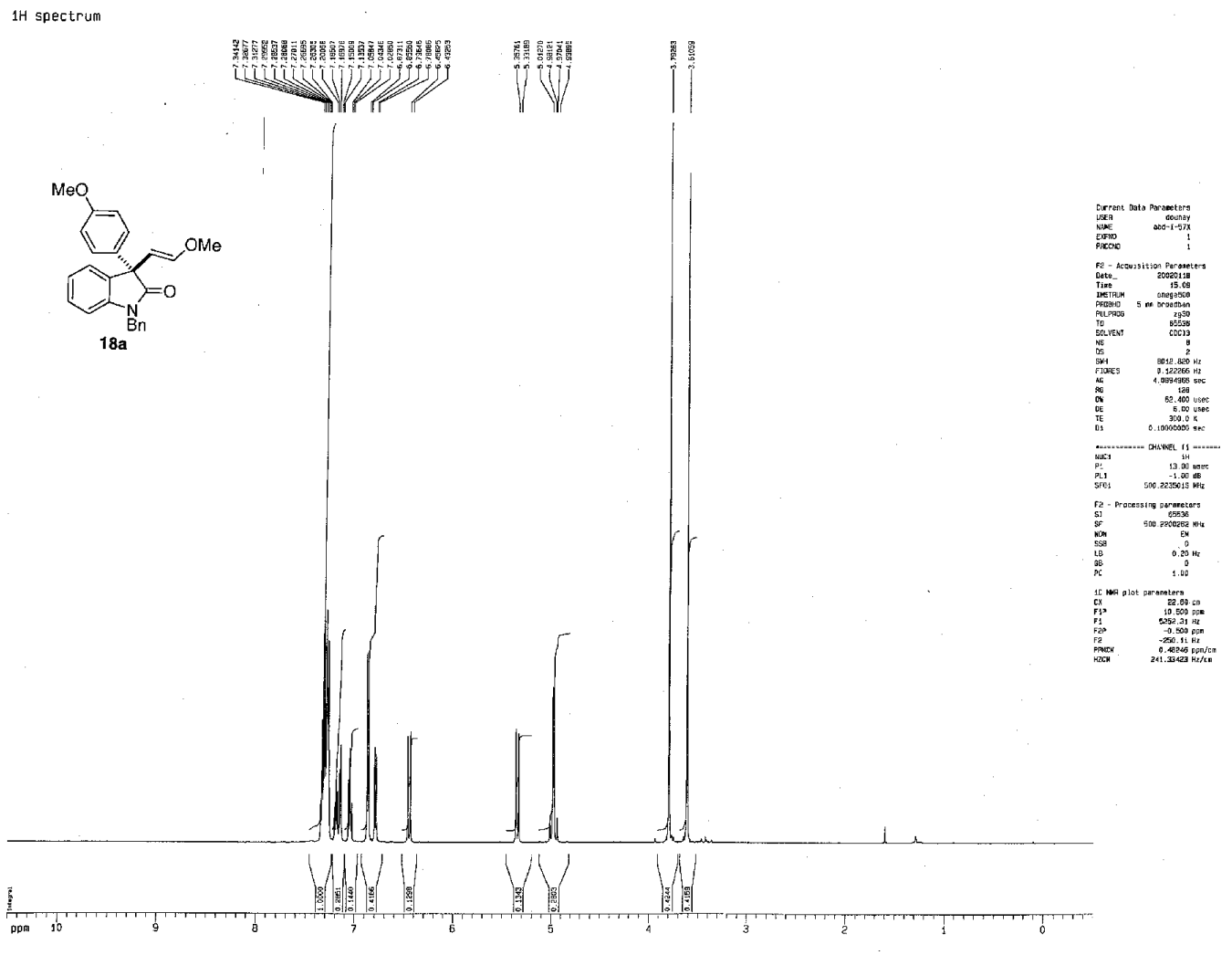

$13 \mathrm{C}$ spectrum with $1 H$ decoupling

s
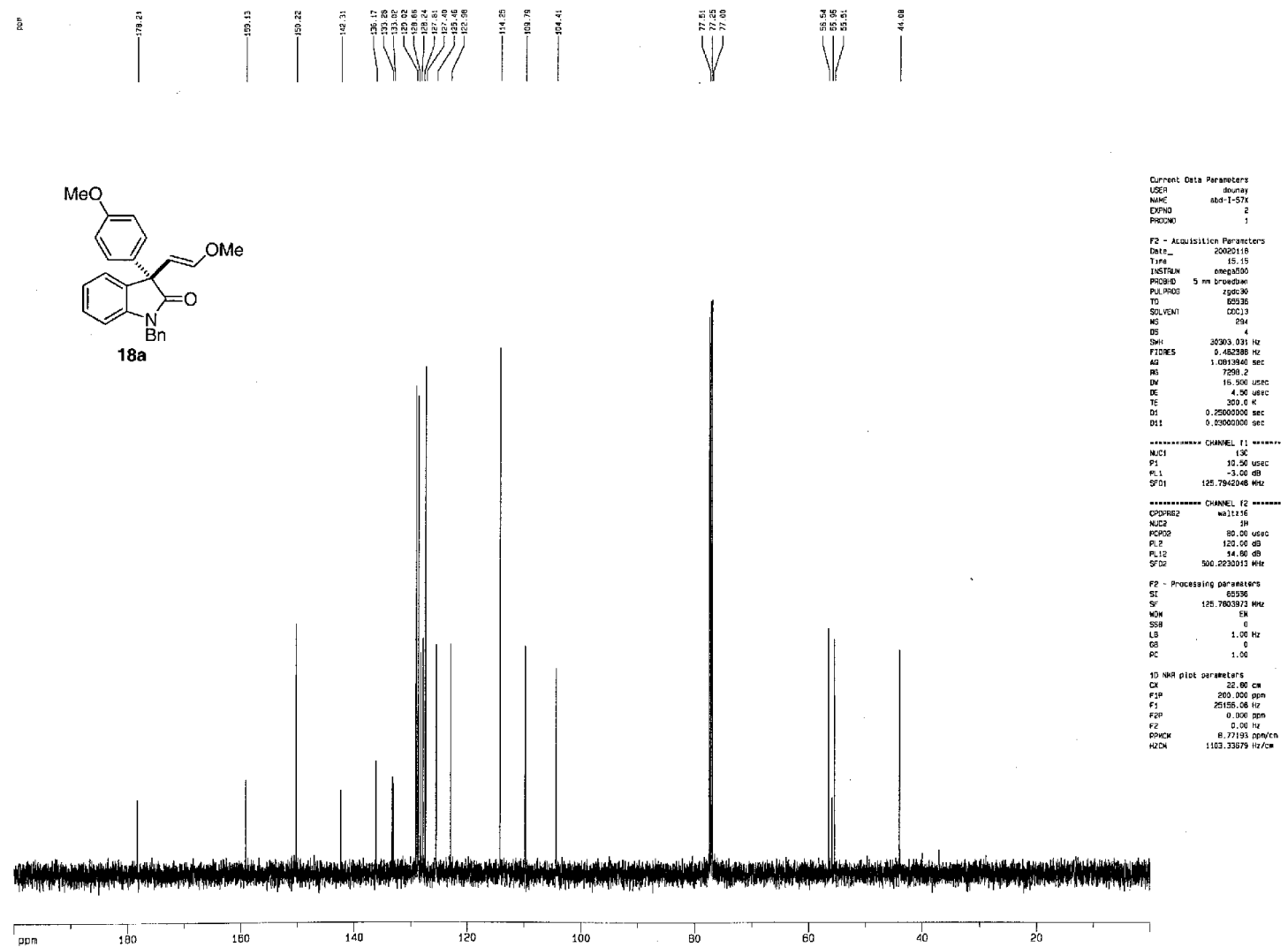
Supporting Information January 28, 2003 Dounay, Hatanaka, Kodanko, Oestreich, Overman*, Pfeifer and Weiss

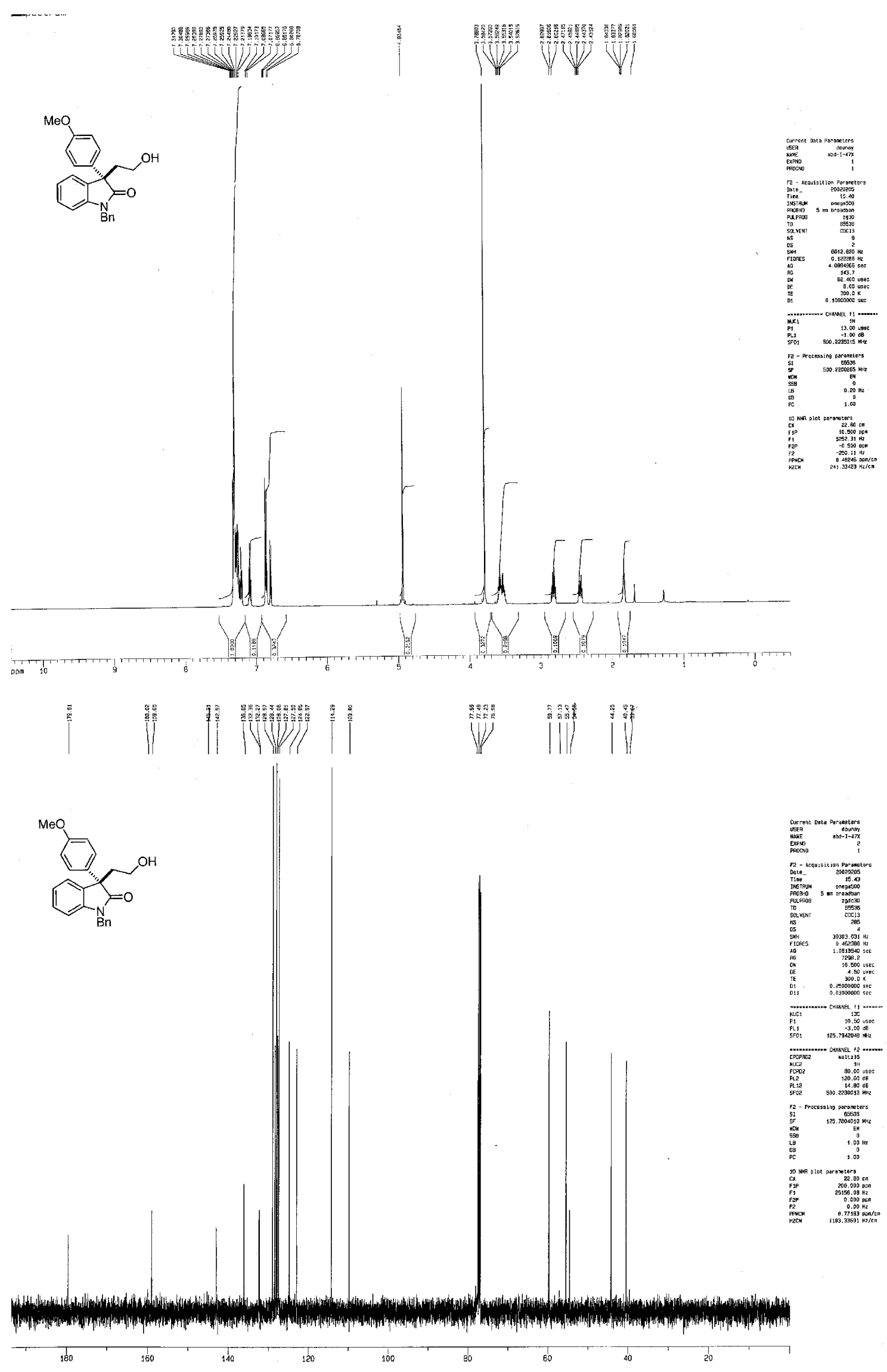


Supporting Information January 28, 2003 Dounay, Hatanaka, Kodanko, Oestreich, Overman*, Pfeifer and Weiss

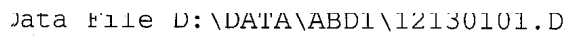

anisolyl system

70:30 Hex/IPA, $1 \mathrm{~mL} / \mathrm{min}$

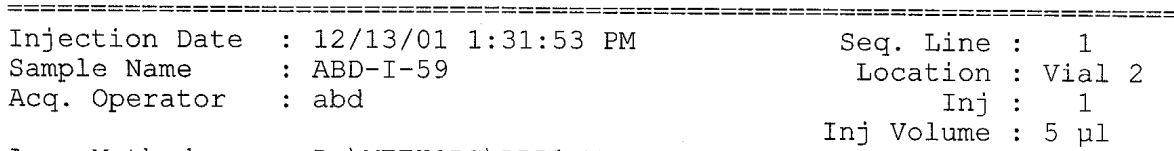

Acq. Method : D: $\backslash$ METHODS $\backslash A B D 1 . M$

Last changed : 11/28/01 1:16:24 PM by abd

Analysis Method : D: \METHODS $\backslash E J V \backslash 3 . M$

Last changed : 9/1/02 12:38:56 PM by EJV

$10 \%$ IPA-Hexanes

$1 \mathrm{~mL} / \mathrm{min}$;

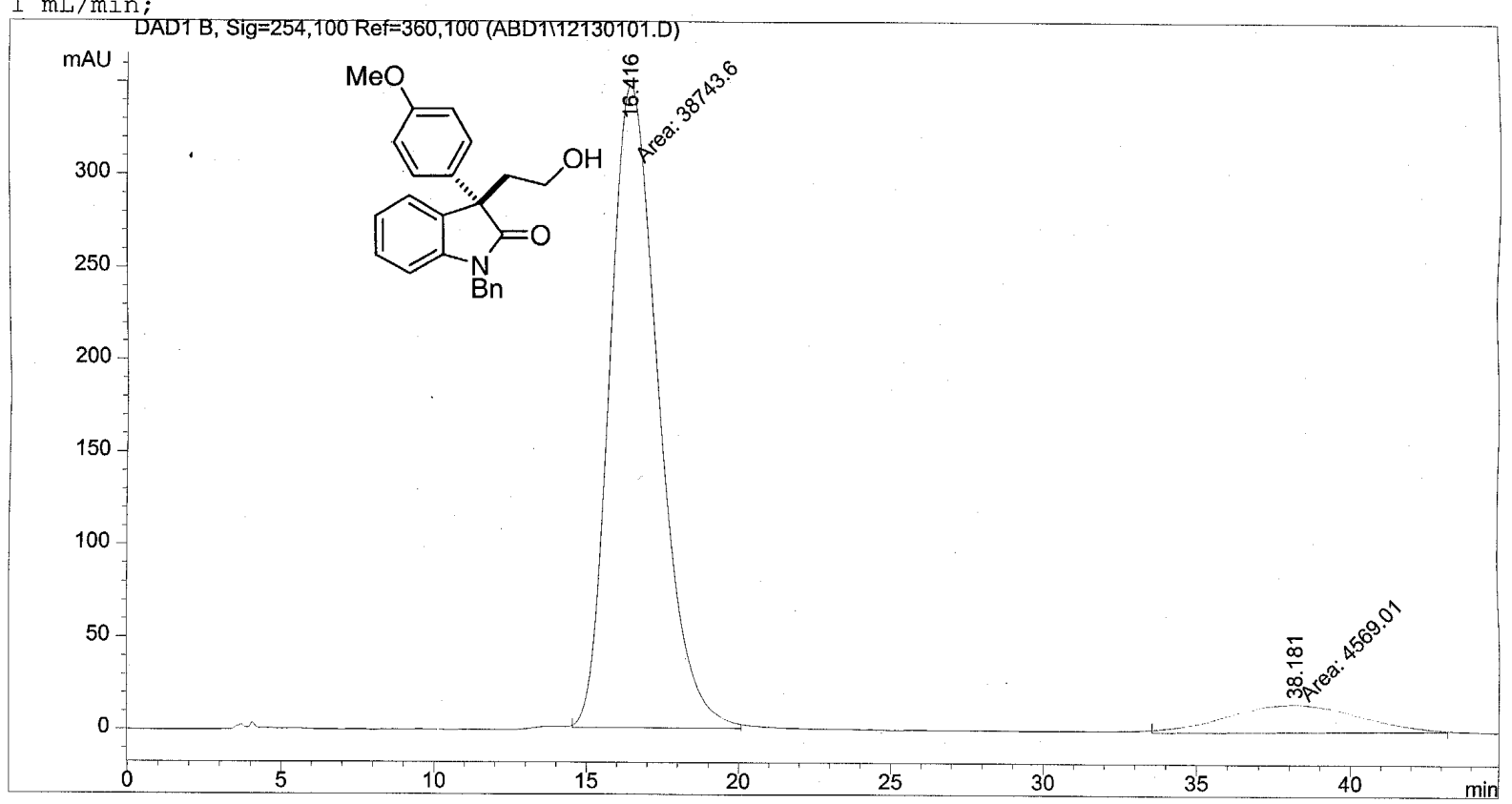

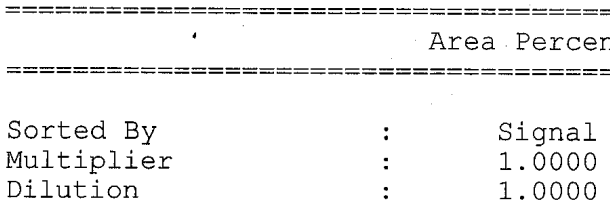

Signal 1: DAD1 B, Sig=254,100 Ref=360,100

\begin{tabular}{|c|c|c|c|c|c|c|}
\hline $\begin{array}{c}\text { Peak } \\
\#\end{array}$ & $\begin{array}{c}\text { RetTime } \\
\text { [min] }\end{array}$ & Type & $\begin{array}{l}\text { Width } \\
\text { [min] }\end{array}$ & $\begin{array}{c}\text { Area } \\
{\left[\mathrm{mAU}{ }^{*} \mathrm{~s}\right]}\end{array}$ & $\begin{array}{l}\text { Height } \\
\text { [mAU] }\end{array}$ & $\begin{array}{c}\text { Area } \\
\frac{\circ}{0}\end{array}$ \\
\hline-- & ------ & r & ------- & |-------- & -------- & -------- \\
\hline 1 & 16.416 & $\mathrm{MM}$ & 1.8601 & $3.87436 \mathrm{e} 4$ & 347.14801 & 89.4511 \\
\hline 2 & 38.181 & MM & 5.0868 & 4569.00928 & 14.97012 & 10.5489 \\
\hline
\end{tabular}

Totals : $\quad 4.33126 e 4 \quad 362.11813$

Results obtained with enhanced integrator!

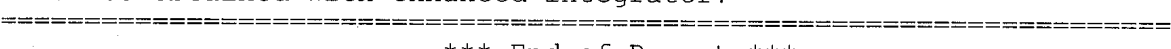

$\star \star \star$ End of Report $\star \star \star$ 
Supporting Information January 28, 2003 Dounay, Hatanaka, Kodanko, Oestreich, Overman*, Pfeifer and Weiss

IH spectrum

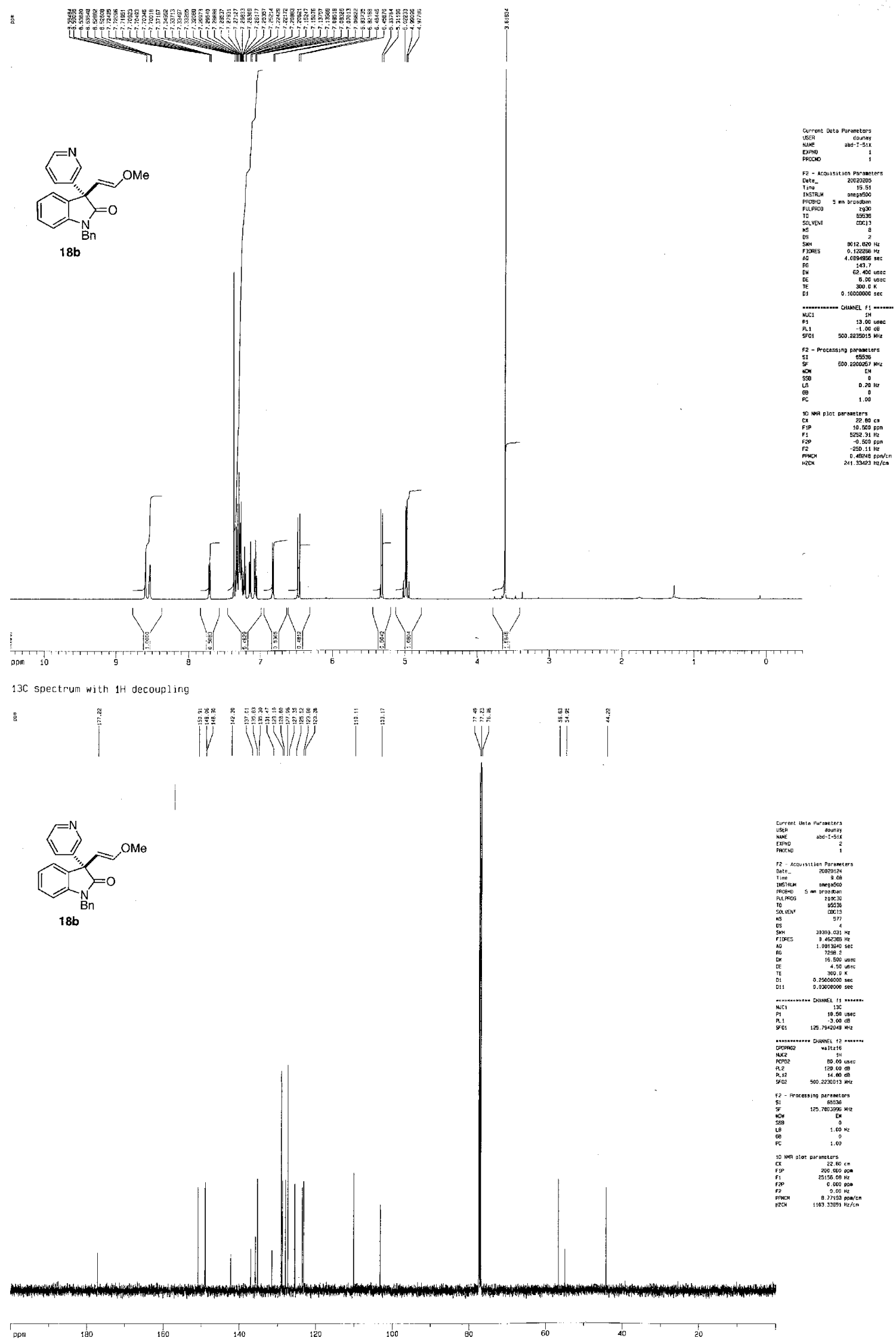


Supporting Information January 28, 2003 Dounay, Hatanaka, Kodanko, Oestreich, Overman*, Pfeifer and Weiss
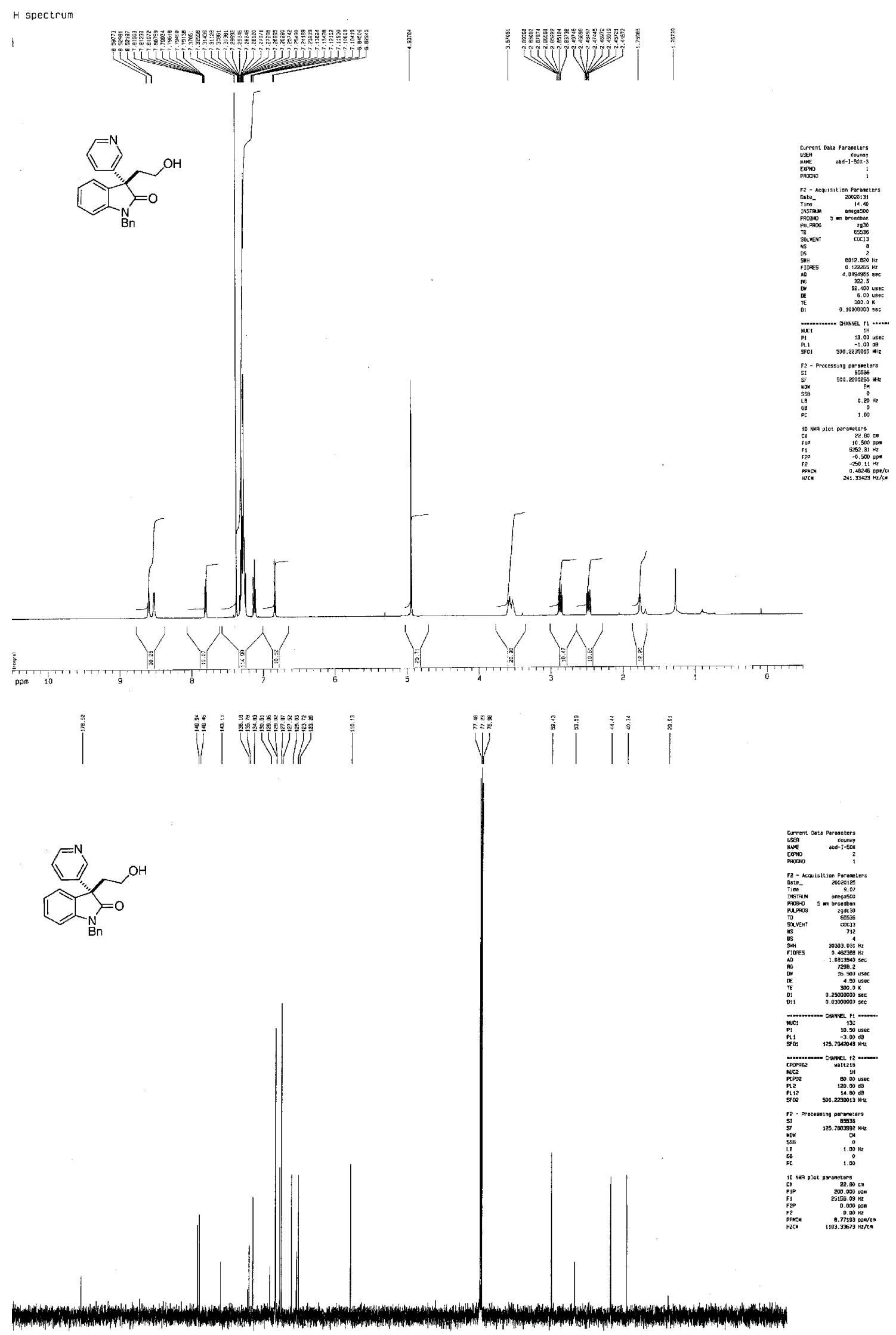

180

${ }_{150}^{1}$

940

120

100

вo 
Supporting Information January 28, 2003 Dounay, Hatanaka, Kodanko, Oestreich, Overman*, Pfeifer and Weiss

Jata File D: \DATA \ABD1 \12070101.D

Sample Name: abd-i-50

pyridinyl product

85:15 Hexanes:IPA, OJ column, $1 \mathrm{~mL} / \mathrm{min}$
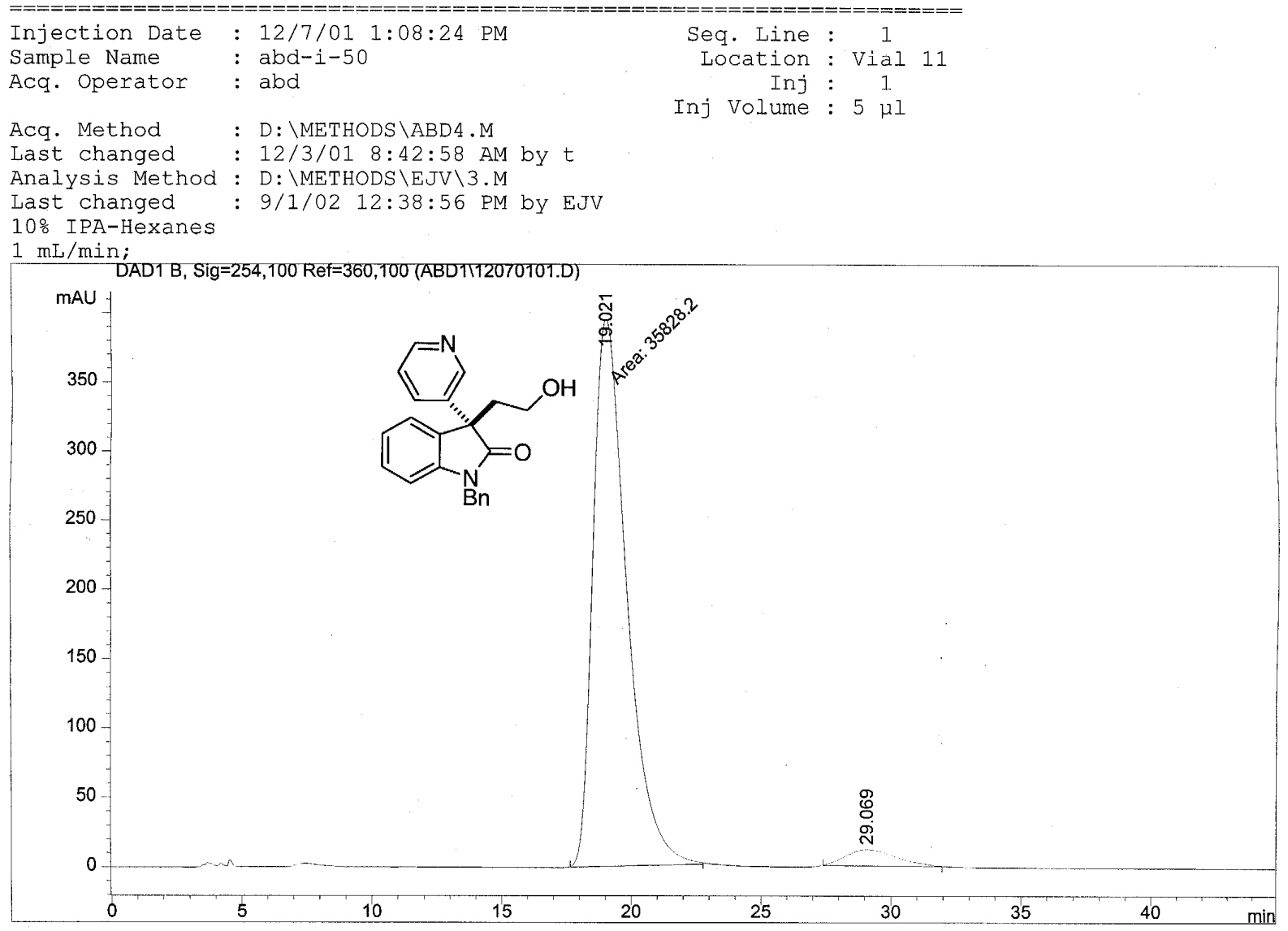

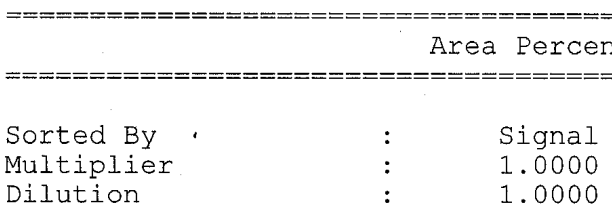

Signal 1: DAD1 B, Sig=254,100 Ref $=360,100$

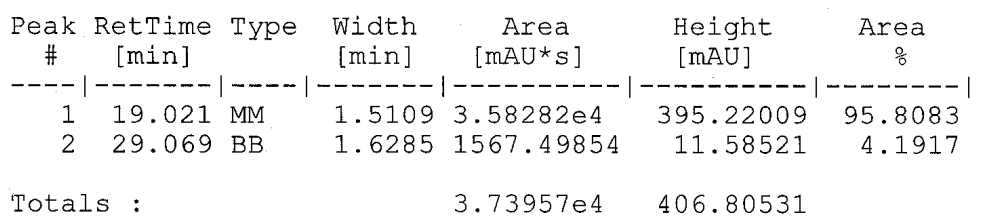

Results obtained with enhanced integrator! 
Supporting Information January 28, 2003 Dounay, Hatanaka, Kodanko, Oestreich, Overman*, Pfeifer and Weiss

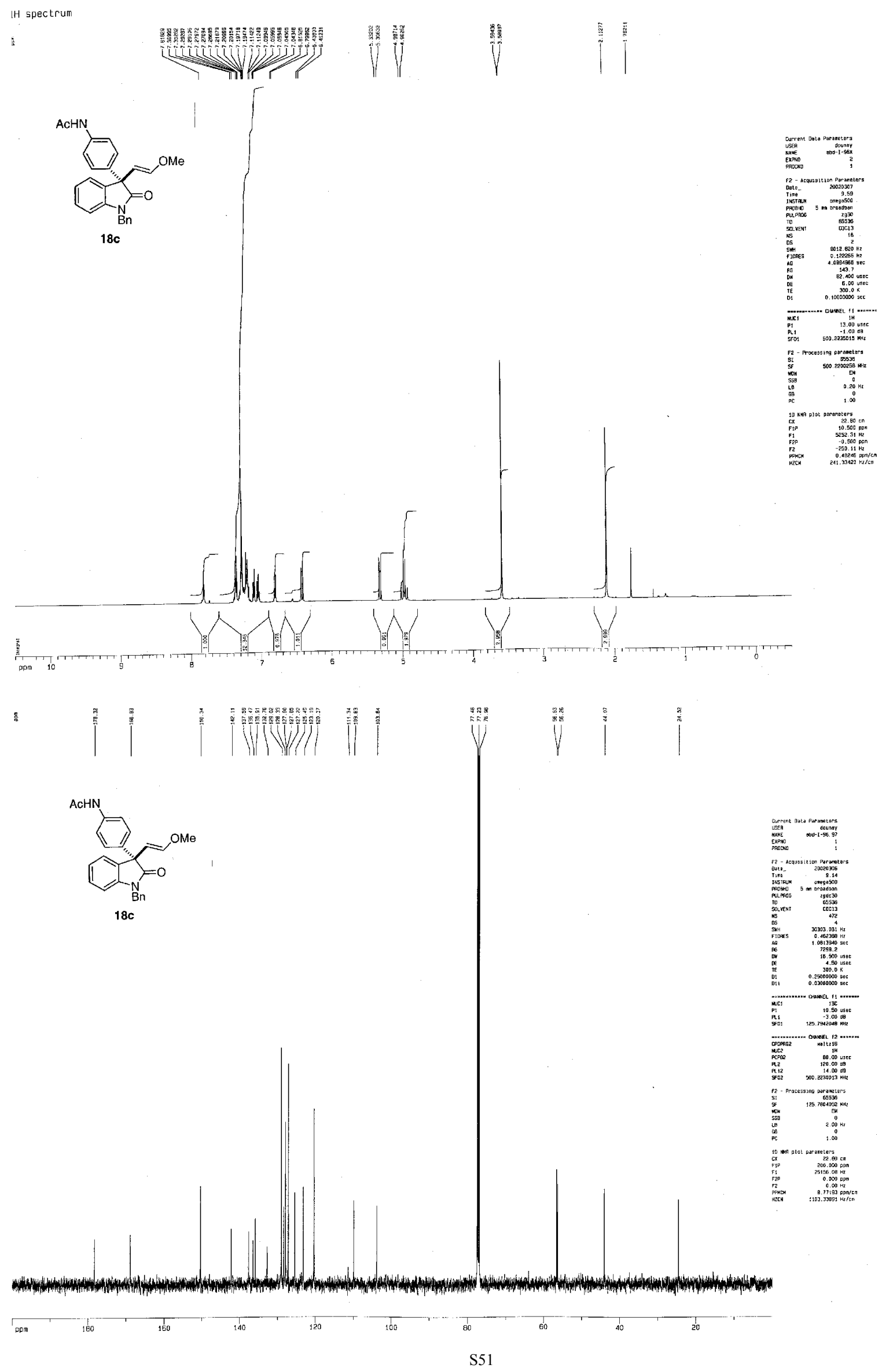


Supporting Information January 28, 2003 Dounay, Hatanaka, Kodanko, Oestreich, Overman*, Pfeifer and Weiss

Data File D: \DATA \ABD1\01150203.D

Sample Name: abd-I-9

acetamide enol ether

75:25 Hex/IPA, AS column, $1 \mathrm{ml} / \mathrm{min}$

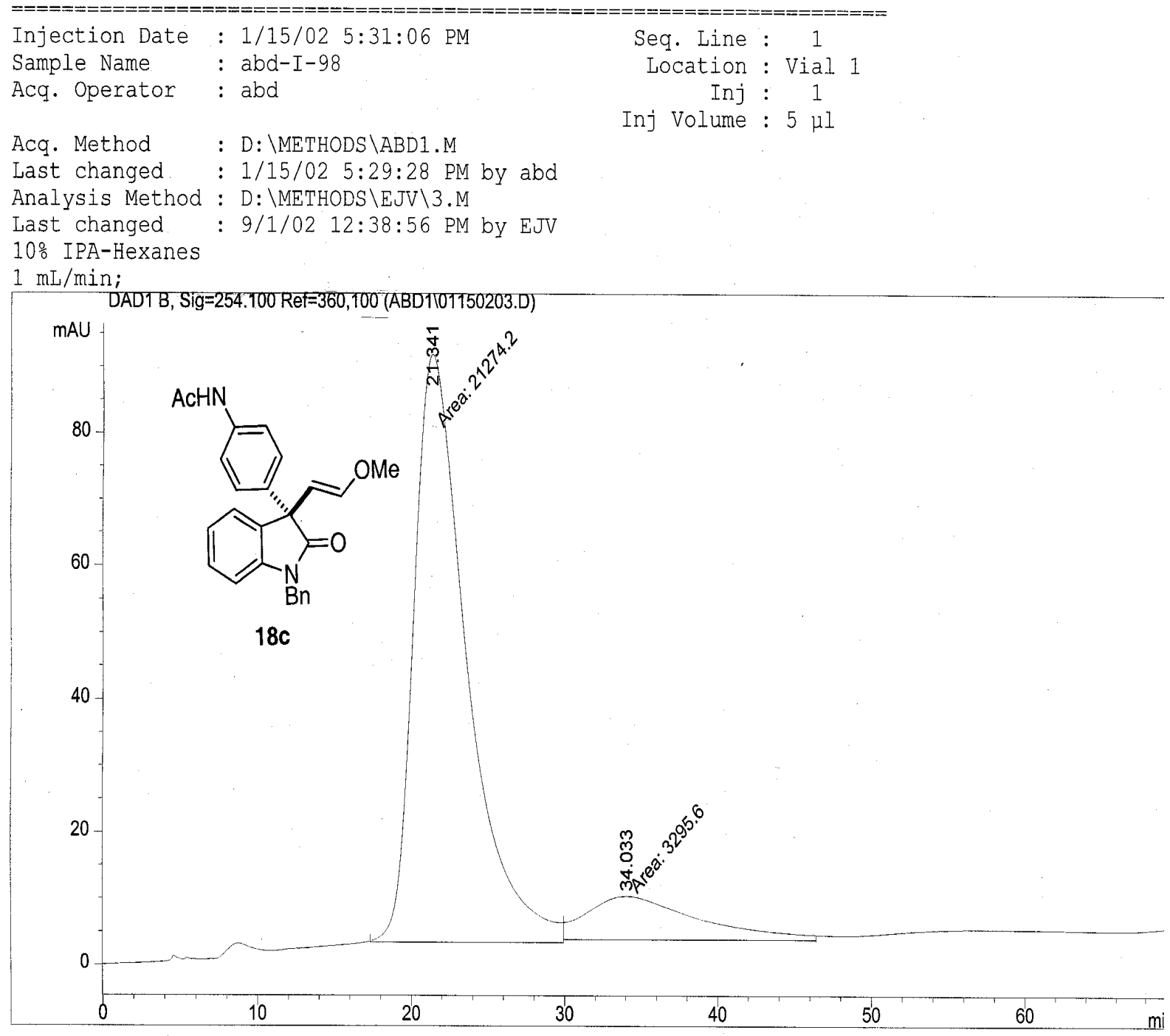

\begin{tabular}{|c|c|c|}
\hline \multicolumn{3}{|c|}{ Area Percen } \\
\hline$= \pm=$ & & r \\
\hline Sorted By & : & Signal \\
\hline Multiplier & : & 1.0000 \\
\hline Dilution & : & 1.0000 \\
\hline
\end{tabular}

Signal 1: DAD1 B, Sig=254,100 Ref $=360,100$

\begin{tabular}{|c|c|c|c|c|c|c|}
\hline $\begin{array}{c}\text { Peak } \\
\#\end{array}$ & $\begin{array}{c}\text { RetTime } \\
\text { [min] }\end{array}$ & Type & $\begin{array}{c}\text { Width } \\
\text { [min] }\end{array}$ & $\begin{array}{c}\text { Area } \\
{\left[\mathrm{mAU}{ }^{*} \mathrm{~S}\right]}\end{array}$ & $\begin{array}{l}\text { Height } \\
\text { [mAU] }\end{array}$ & $\begin{array}{c}\text { Area } \\
\frac{\circ}{0}\end{array}$ \\
\hline & & & & --- & & \\
\hline 1 & 21 & MM & 4.0077 & $2.12742 \mathrm{e} 4$ & 88.47134 & 68 \\
\hline 2 & 34.1 & $\mathrm{MIN}$ & 9 & 329 & 1954 & 32 \\
\hline
\end{tabular}

Totals : $\quad 2.45698 \mathrm{e} 4 \quad 94.99087$

Results obtained with enhanced integrator! 
Supporting Information January 28, 2003 Dounay, Hatanaka, Kodanko, Oestreich, Overman*, Pfeifer and Weiss
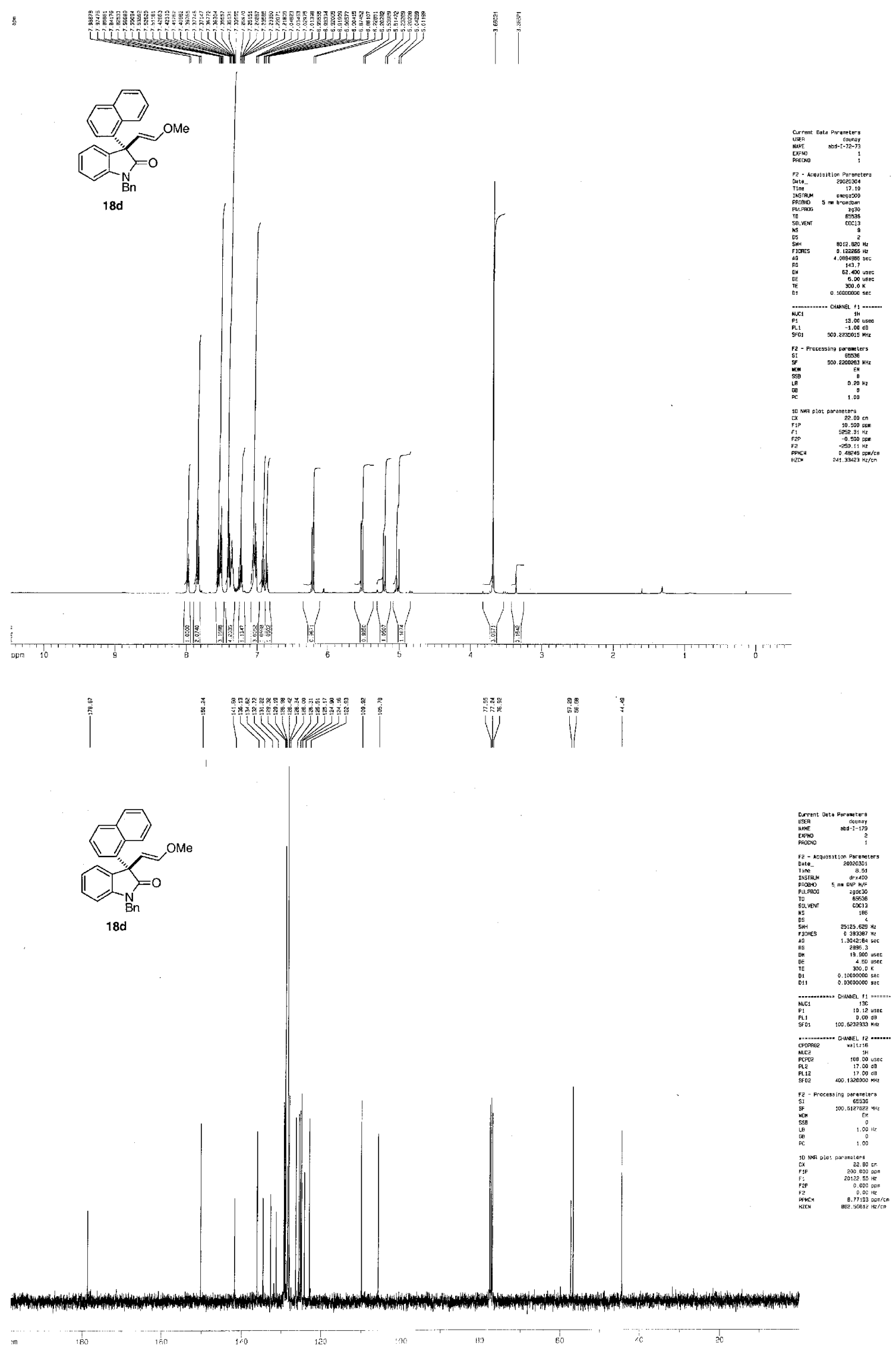
Supporting Information January 28, 2003 Dounay, Hatanaka, Kodanko, Oestreich, Overman*, Pfeifer and Weiss

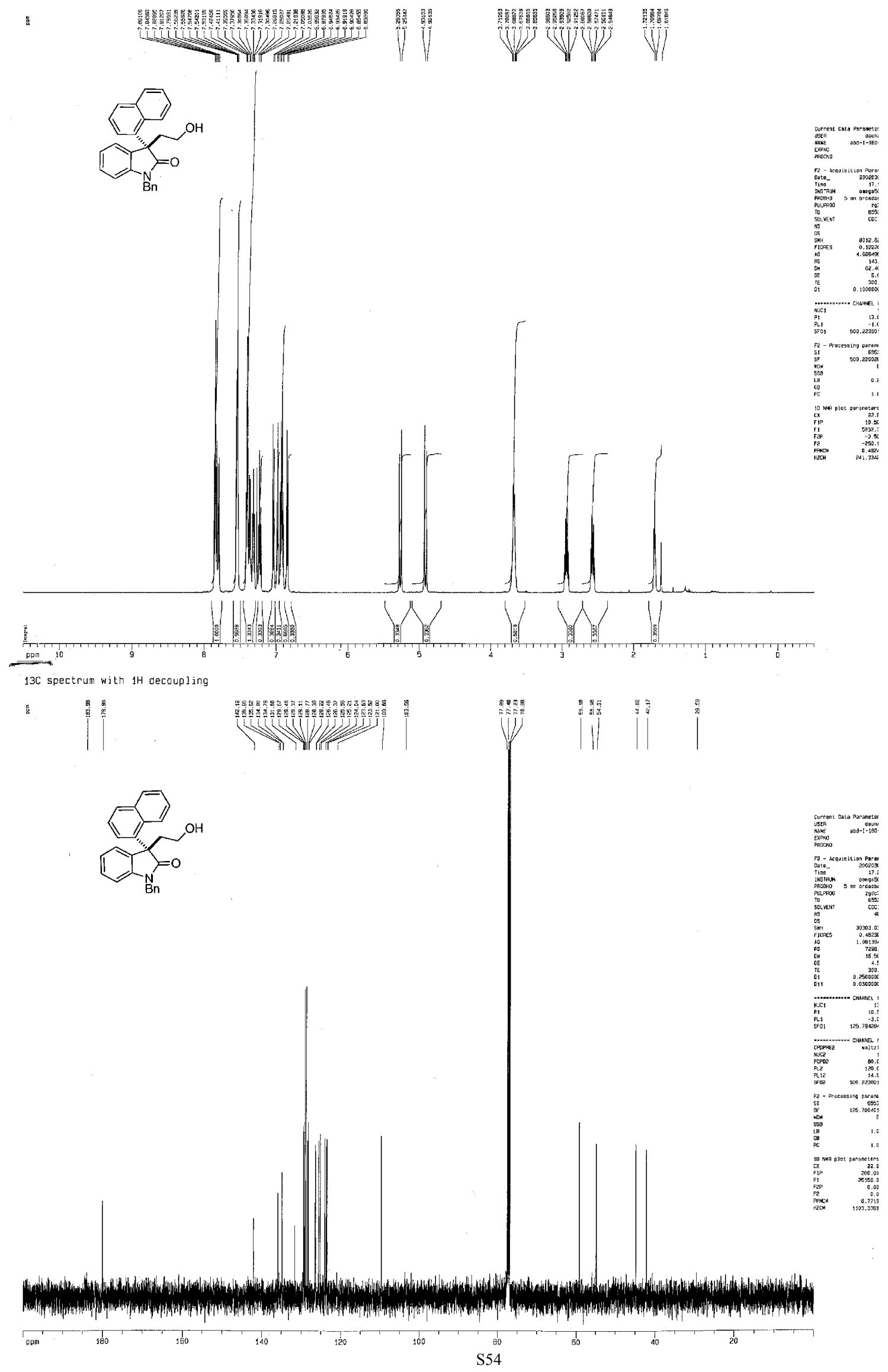


Supporting Information January 28, 2003 Dounay, Hatanaka, Kodanko, Oestreich, Overman*, Pfeifer and Weiss

OD-H, 85:15 Hex/IPA $1 \mathrm{~mL} / \mathrm{min}$
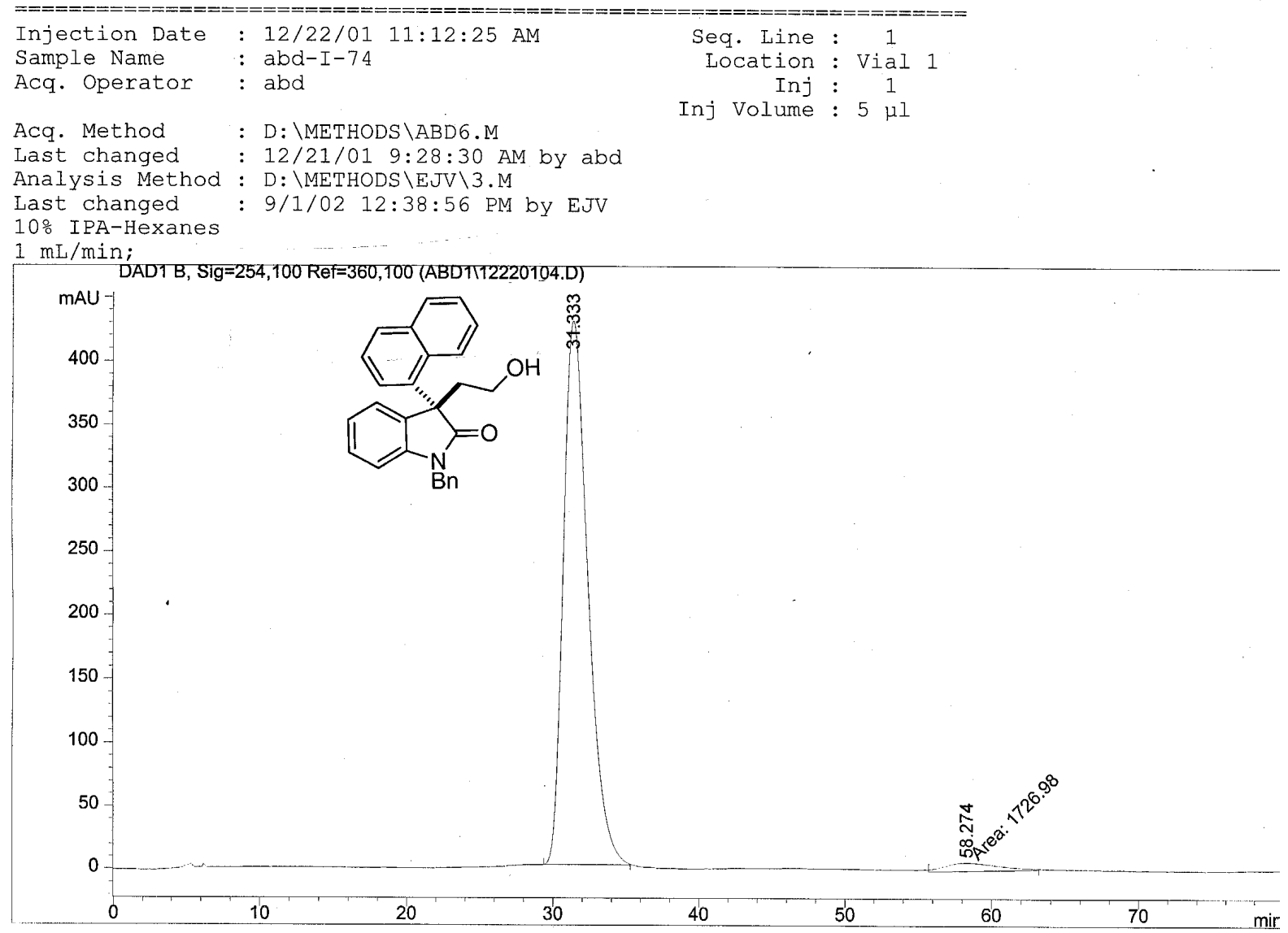

\begin{tabular}{|c|c|c|}
\hline \multicolumn{3}{|c|}{$\begin{array}{l}\text { Area Percen } \\
=========\end{array}$} \\
\hline Sorted By & : & Signal \\
\hline Multiplier & : & 1.0000 \\
\hline Dilution & : & 1.0000 \\
\hline
\end{tabular}

Signal 1: DAD1 B, Sig=254, 100 Ref $=360,100$

\begin{tabular}{|c|c|c|c|c|c|c|}
\hline $\begin{array}{c}\text { Peak } \\
\#\end{array}$ & $\begin{array}{c}\text { RetTime } \\
\text { [min] }\end{array}$ & Type & $\begin{array}{c}\text { width } \\
\text { [min] }\end{array}$ & $\begin{array}{c}\text { Area } \\
{\left[\mathrm{mAU}^{*} \mathrm{~s}\right]}\end{array}$ & $\begin{array}{c}\text { Height } \\
{[\mathrm{mAU}]}\end{array}$ & $\begin{array}{c}\text { Area } \\
\frac{g}{\delta}\end{array}$ \\
\hline---- & $---\cdots--1$ & & -- & $1----$ & --------1 & ------1 \\
\hline 1 & 31.333 & $\mathrm{BB}$ & 1.7627 & $5.01476 \mathrm{e} 4$ & 428.09079 & 96.6709 \\
\hline 2 & 58.274 & MM & 4.4195 & 1726.97705 & 6.51265 & 3.3291 \\
\hline Tota] & : & & & $5.18746 \mathrm{e} 4$ & 434.60344 & \\
\hline
\end{tabular}

Results obtained with enhanced integrator! 
Supporting Information January 28, 2003 Dounay, Hatanaka, Kodanko, Oestreich, Overman*, Pfeifer and Weiss

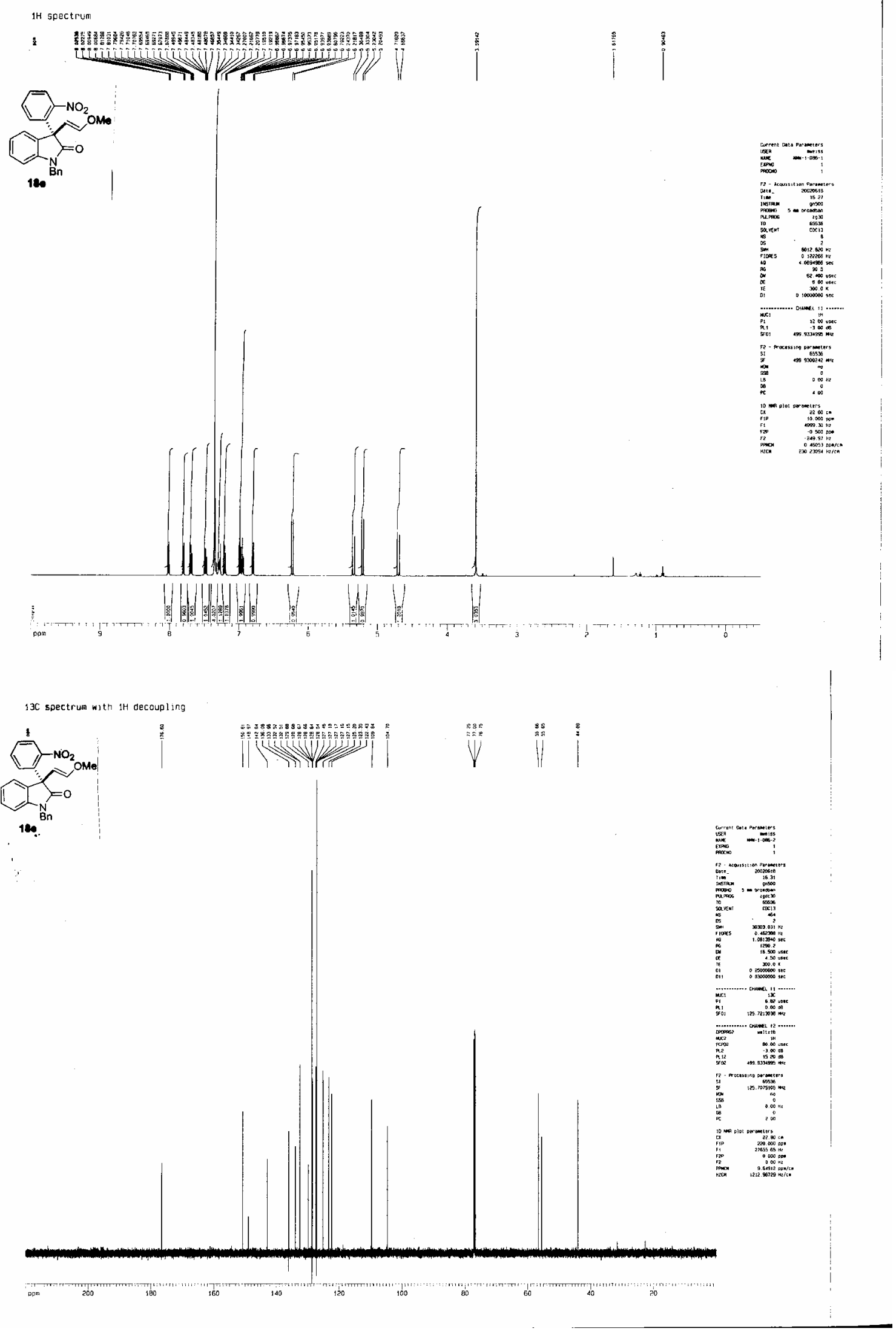



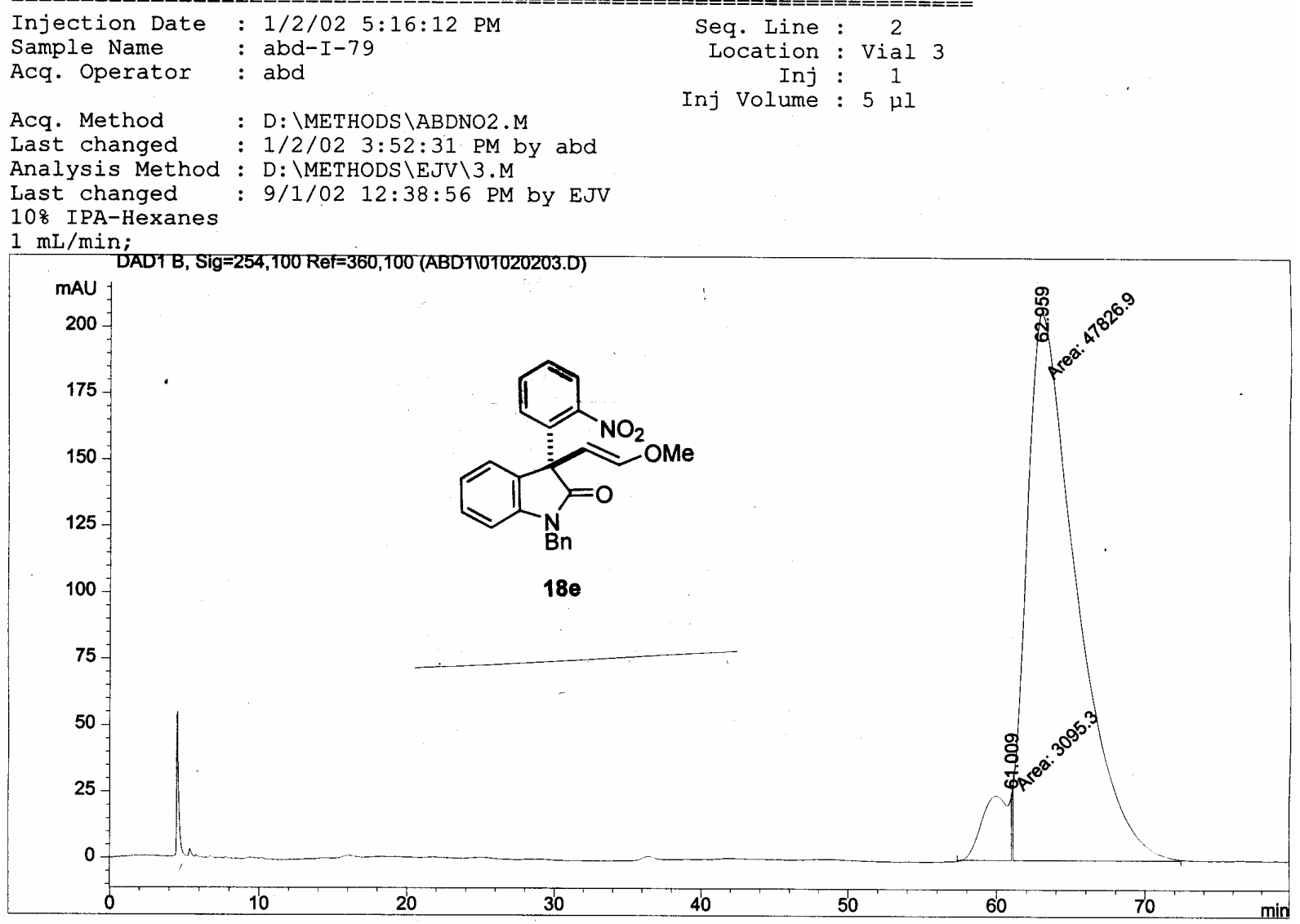

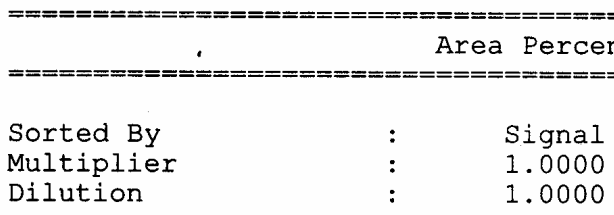

Signal 1: DAD1 B, Sig=254,100 Ref $=360,100$

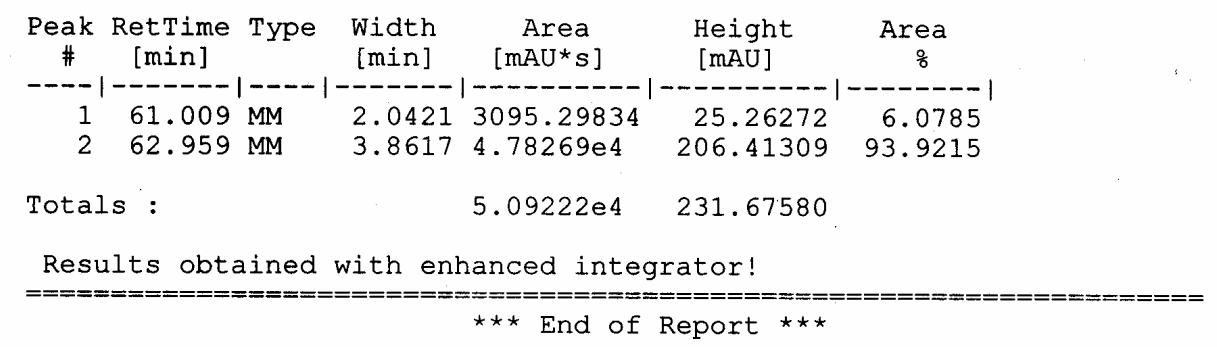


Supporting Information January 28, 2003 Dounay, Hatanaka, Kodanko, Oestreich, Overman*, Pfeifer and Weiss

MO-II-29 (500 MHz) Heck Product

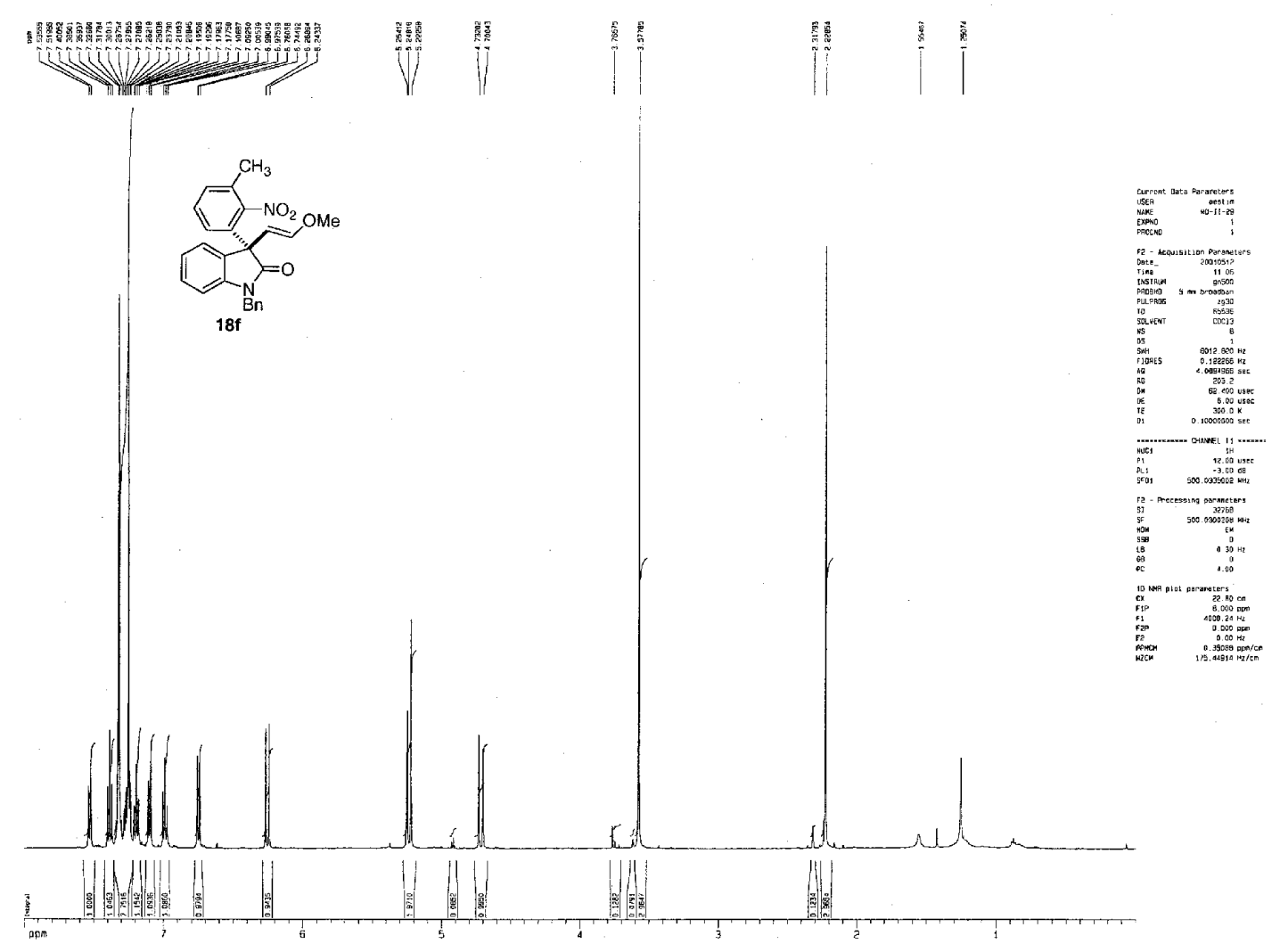

MD-11-29 (125 MHz) Heck Product

忘
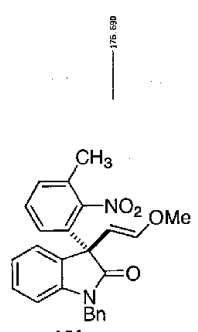

$18 f$

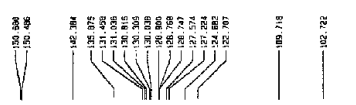

Wij
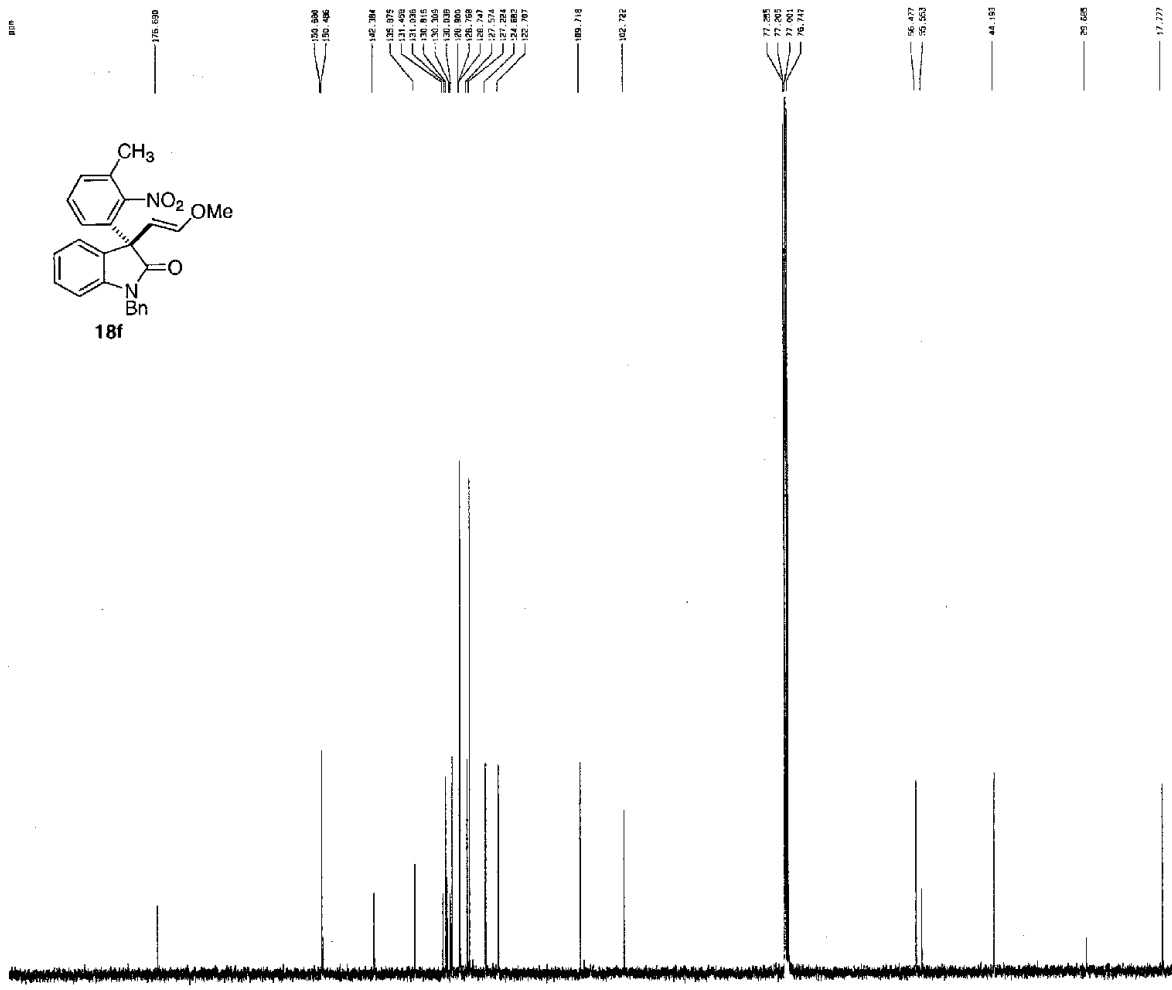

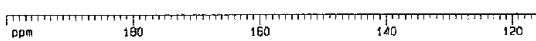

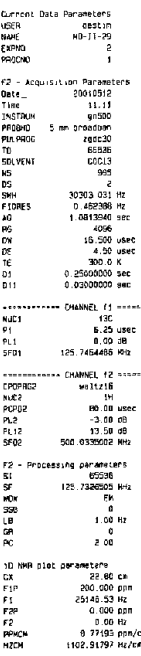


Supporting Information January 28, 2003 Dounay, Hatanaka, Kodanko, Oestreich, Overman*, Pfeifer and Weiss

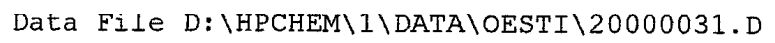

Heck product with ortho-nitro substituent
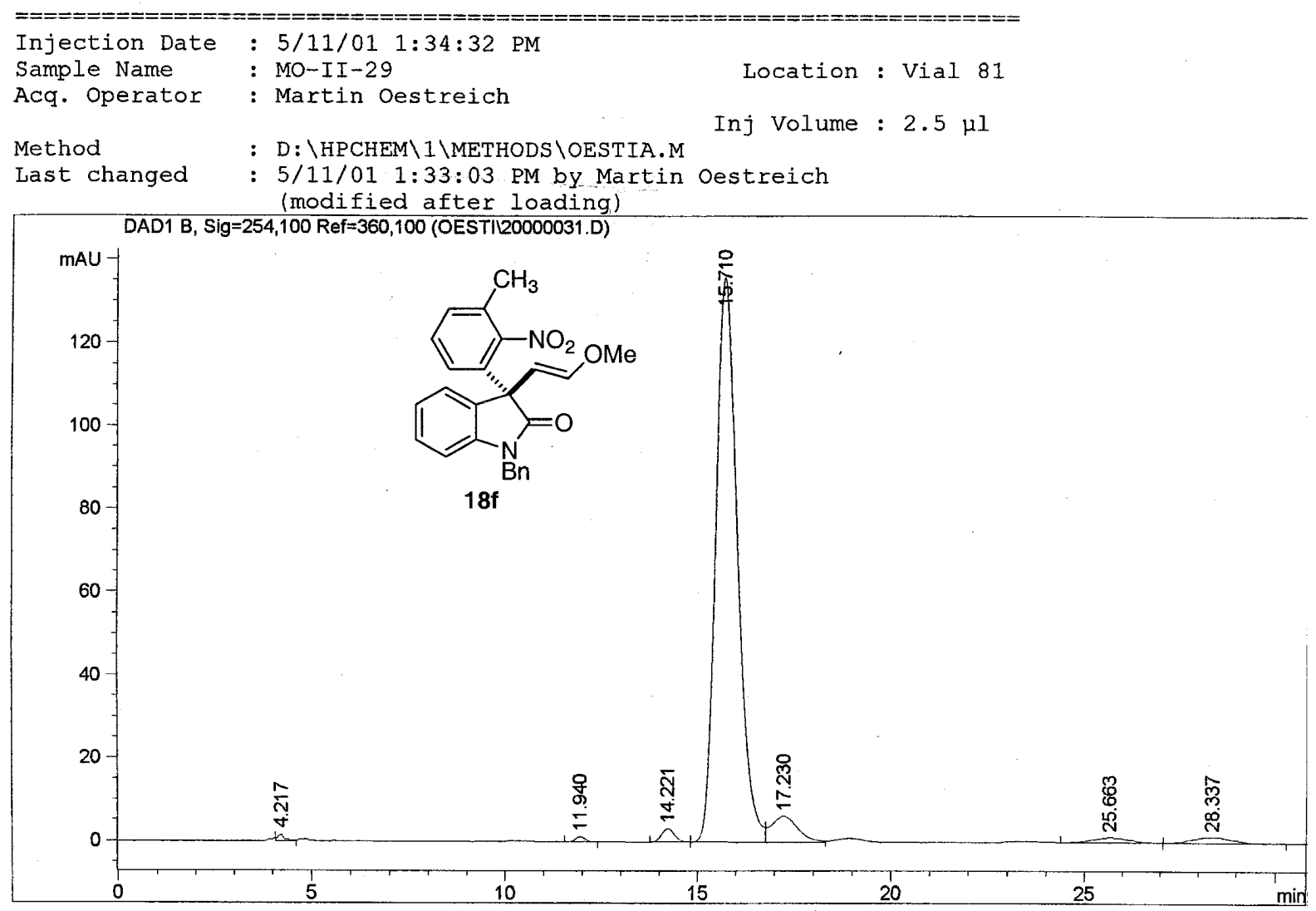

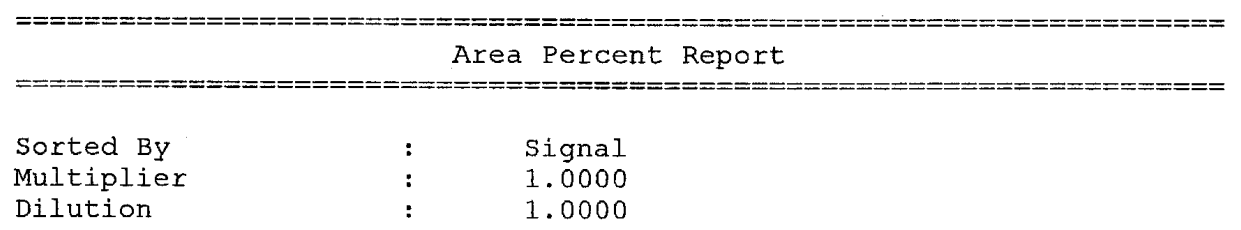

Signal 1: DADI B, Sig=254,100 Ref $=360,100$

\begin{tabular}{|c|c|c|c|c|c|c|}
\hline $\begin{array}{c}\text { Peak } \\
\#\end{array}$ & $\begin{array}{l}\text { RetTime } \\
\text { [min] }\end{array}$ & Type & $\begin{array}{l}\text { Width } \\
{[\min ]}\end{array}$ & $\begin{array}{c}\text { Area } \\
{\left[\mathrm{mAU}^{\star} \mathrm{s}\right]}\end{array}$ & $\begin{array}{l}\text { Height } \\
{[\mathrm{mAU}]}\end{array}$ & $\begin{array}{c}\text { Area } \\
\frac{8}{8}\end{array}$ \\
\hline 1 & 4.217 & VV & 0.1610 & 19.03525 & 1.55255 & 0.3189 \\
\hline 2 & 11.940 & VP & 0 . & & & 0. \\
\hline 3 & 14.221 & BV & 0.3 & 76.16884 & 114 & 1.2763 \\
\hline 4 & 15.710 & VV & 0.6052 & 5349.29004 & 135.90143 & 89.6304 \\
\hline 5 & 17.230 & VV & 0.7247 & 296.09927 & 6.13944 & 4.9613 \\
\hline 6 & 25.663 & VV & 0.8628 & 92.08887 & 1.29311 & 1.5430 \\
\hline 7 & 28.337 & VP & 0.9461 & 114.58689 & 1.49851 & 1.9200 \\
\hline . & & & & 5968.16770 & 82040 & \\
\hline
\end{tabular}

Results obtained with enhanced integrator! 

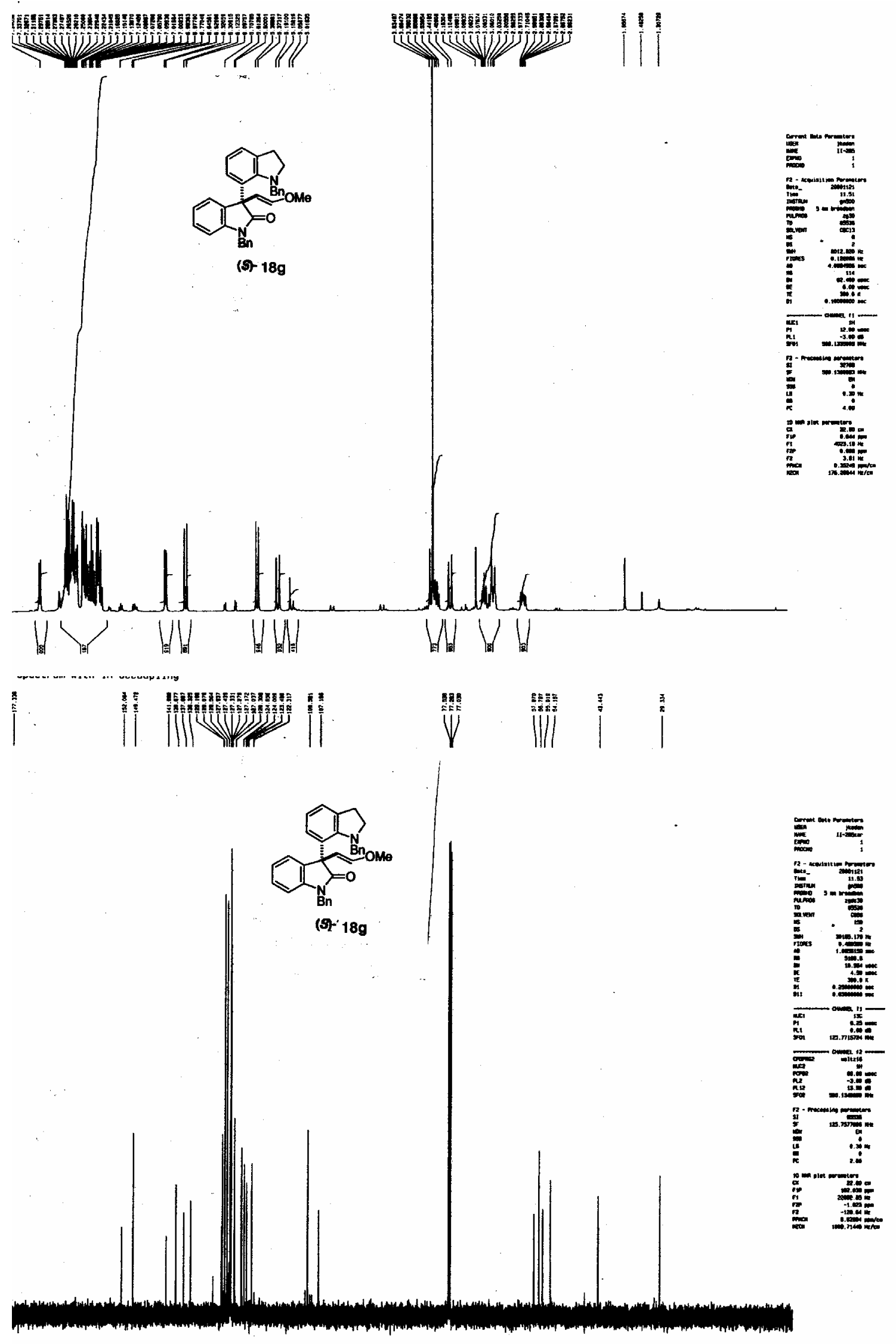

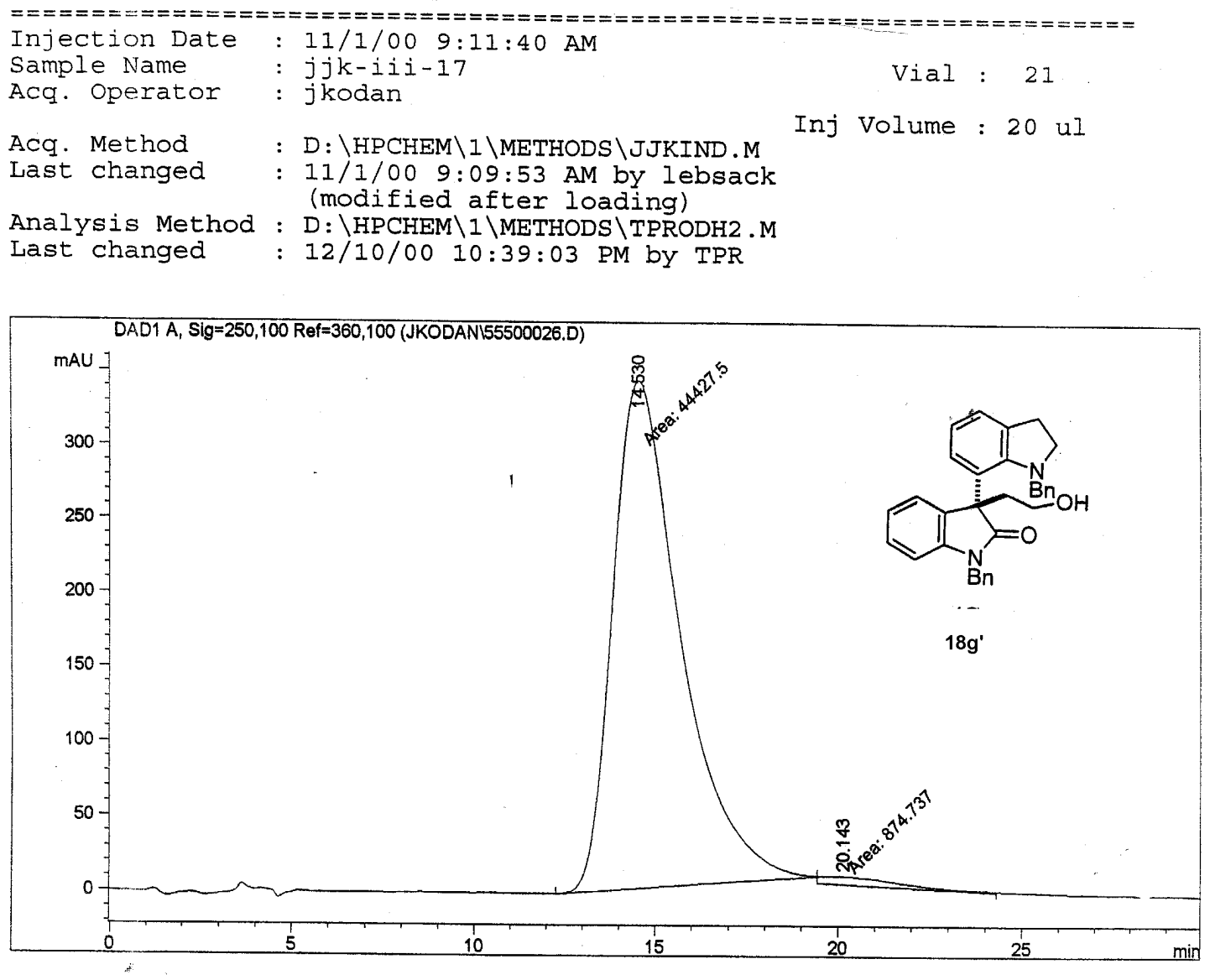

\begin{tabular}{|c|c|c|}
\hline Sorted EY & : & Signal \\
\hline Multiplier & $:$ & 1.0000 \\
\hline Dilution & : & 1.0000 \\
\hline
\end{tabular}

Signal 1: DAD1 A, Sig $=250,100$ Ref $=360,100$

\begin{tabular}{|c|c|c|c|c|c|c|}
\hline Peak & $\begin{array}{l}\text { RetTime } \\
\text { [min] }\end{array}$ & Type & $\begin{array}{l}\text { Width } \\
\text { [min] }\end{array}$ & $\begin{array}{c}\text { Area } \\
{[\mathrm{mAU} * \mathrm{~s}]}\end{array}$ & $\begin{array}{l}\text { Height } \\
\text { [mAU] }\end{array}$ & $\begin{array}{c}\text { Area } \\
\frac{\circ}{0}\end{array}$ \\
\hline $\begin{array}{l}1 \\
2\end{array}$ & $\begin{array}{l}14.530 \\
20.143\end{array}$ & $\begin{array}{l}\text { MM } \\
\text { MM }\end{array}$ & $\begin{array}{l}2.1648 \\
2.6704\end{array}$ & $\begin{array}{c}4.44275 e 4 \\
874.73718\end{array}$ & $\begin{array}{r}342.03894 \\
5.45939\end{array}$ & $\begin{array}{r}98.0691 \\
1.9309\end{array}$ \\
\hline Tota & & & & $4.53022 e 4$ & 347 & \\
\hline
\end{tabular}

Results obtained with enhanced integrator!

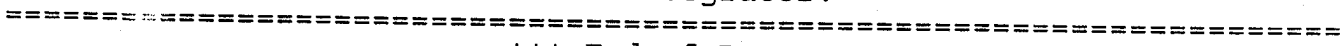
*** End of Report *** 
Supporting Information January 28, 2003 Dounay, Hatanaka, Kodanko, Oestreich, Overman*, Pfeifer and Weiss

$1 \mathrm{H}$ spectrum

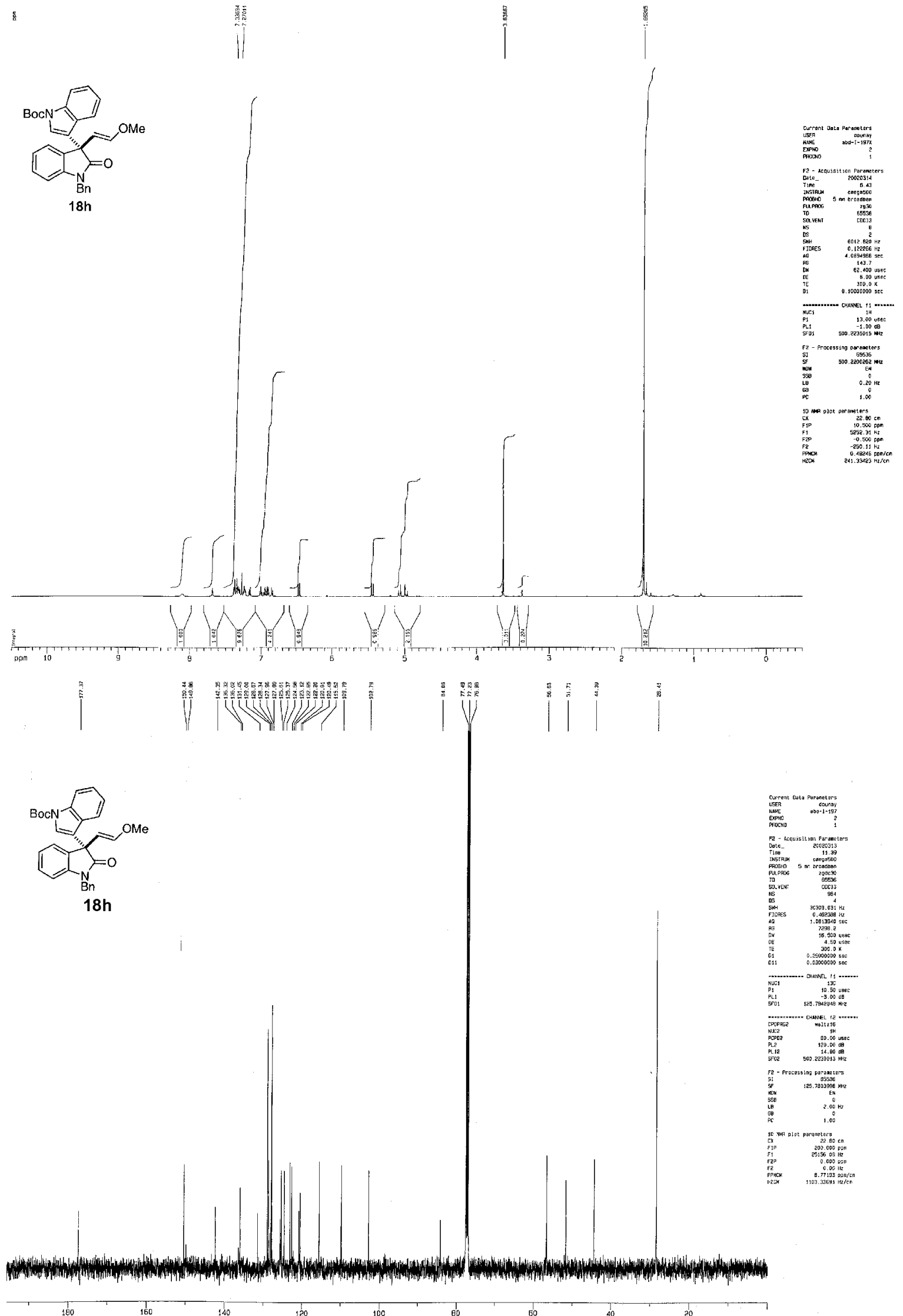


OD-H, $80: 20 \mathrm{Hex} / \mathrm{IPA}, 1 \mathrm{mi} / \mathrm{min}$

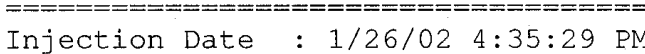

Sample Name : abd-I-118

Acq. Operator : amy

$\begin{array}{rcc}\text { Seq. Line } & : & 1 \\ \text { Location } & : \text { Vial } \\ \text { Inj } & : & 1 \\ \text { Inj Volume } & : 5 \mu l\end{array}$

Acq. Method

: D: \METHODS $\backslash A B D 1 . M$

Inj Volume : $5 \mu 1$

Last changed : 1/24/02 9:51:15 PM by ABD

Analysis Method : D: \METHODS $\backslash$ EJV $\backslash 3 . M$

Last changed : 9/1/02 12:38:56 PM by EJV

$10 \%$ IPA-Hexanes

$1 \mathrm{~mL} / \mathrm{min}$;

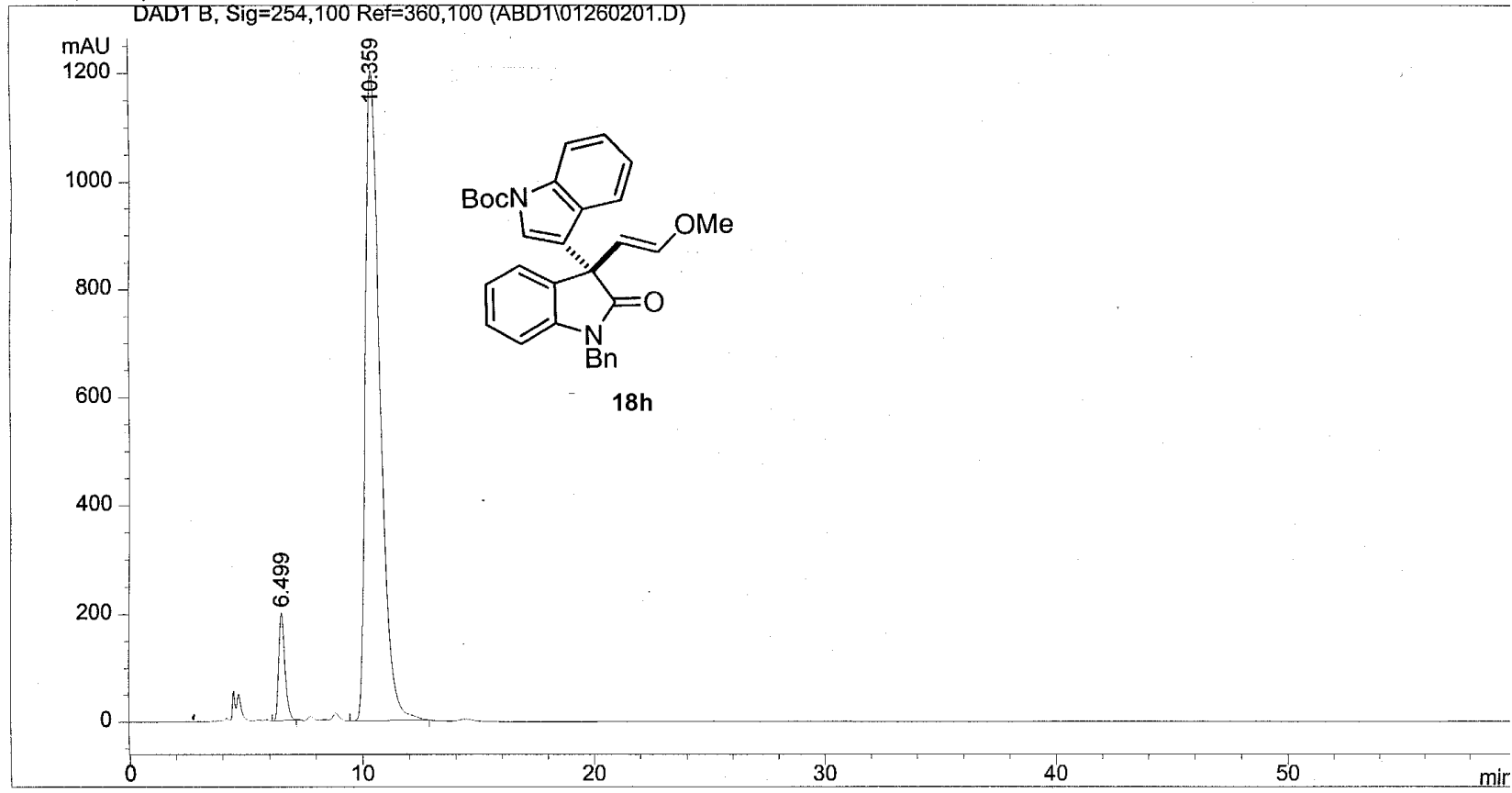

Area Percent Report

$\begin{array}{lll}\text { Sorted By } & : & \text { Signal } \\ \text { Multiplier } & : & 1.0000 \\ \text { Dilution } & : & 1.0000\end{array}$

Signal 1: DAD1 B, Sig=254,100 Ref $=360,100$

\begin{tabular}{|c|c|c|c|c|c|c|}
\hline $\begin{array}{c}\text { Peak } \\
\#\end{array}$ & $\begin{array}{c}\text { RetTime } \\
\text { [min] }\end{array}$ & Type & $\begin{array}{c}\text { Width } \\
\text { [min] }\end{array}$ & $\begin{array}{c}\text { Area } \\
{\left[\mathrm{mAU}{ }^{*} \mathrm{~S}\right]}\end{array}$ & $\begin{array}{l}\text { Height } \\
\text { [mAU] }\end{array}$ & $\begin{array}{c}\text { Area } \\
\frac{\circ}{0}\end{array}$ \\
\hline 1 & 6.499 & VP & 0.2809 & 3682.41333 & 199.93222 & 6.5327 \\
\hline 2 & 10.359 & VB & 0.6728 & $5.26868 \mathrm{e} 4$ & 1203.45642 & 93.4673 \\
\hline ta & & & & $5.63692 \mathrm{e} 4$ & 1403.38864 & \\
\hline
\end{tabular}

Results obtained with enhanced integrator!

$\star * *$ End of Report $* * *$

1 
Supporting Information January 28, 2003 Dounay, Hatanaka, Kodanko, Oestreich, Overman*, Pfeifer and Weiss
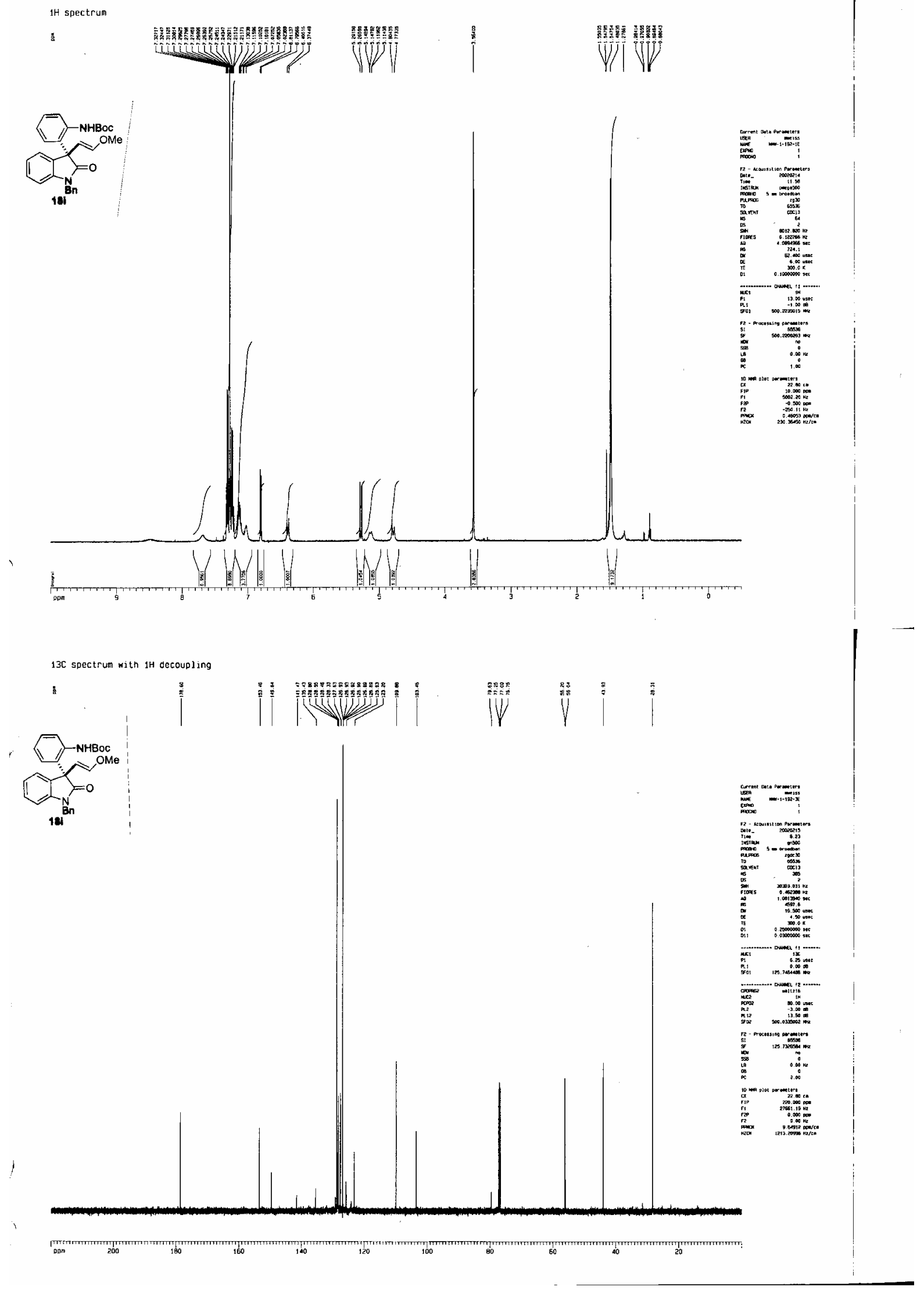
Supporting Information January 28, 2003 Dounay, Hatanaka, Kodanko, Oestreich, Overman*, Pfeifer and Weiss

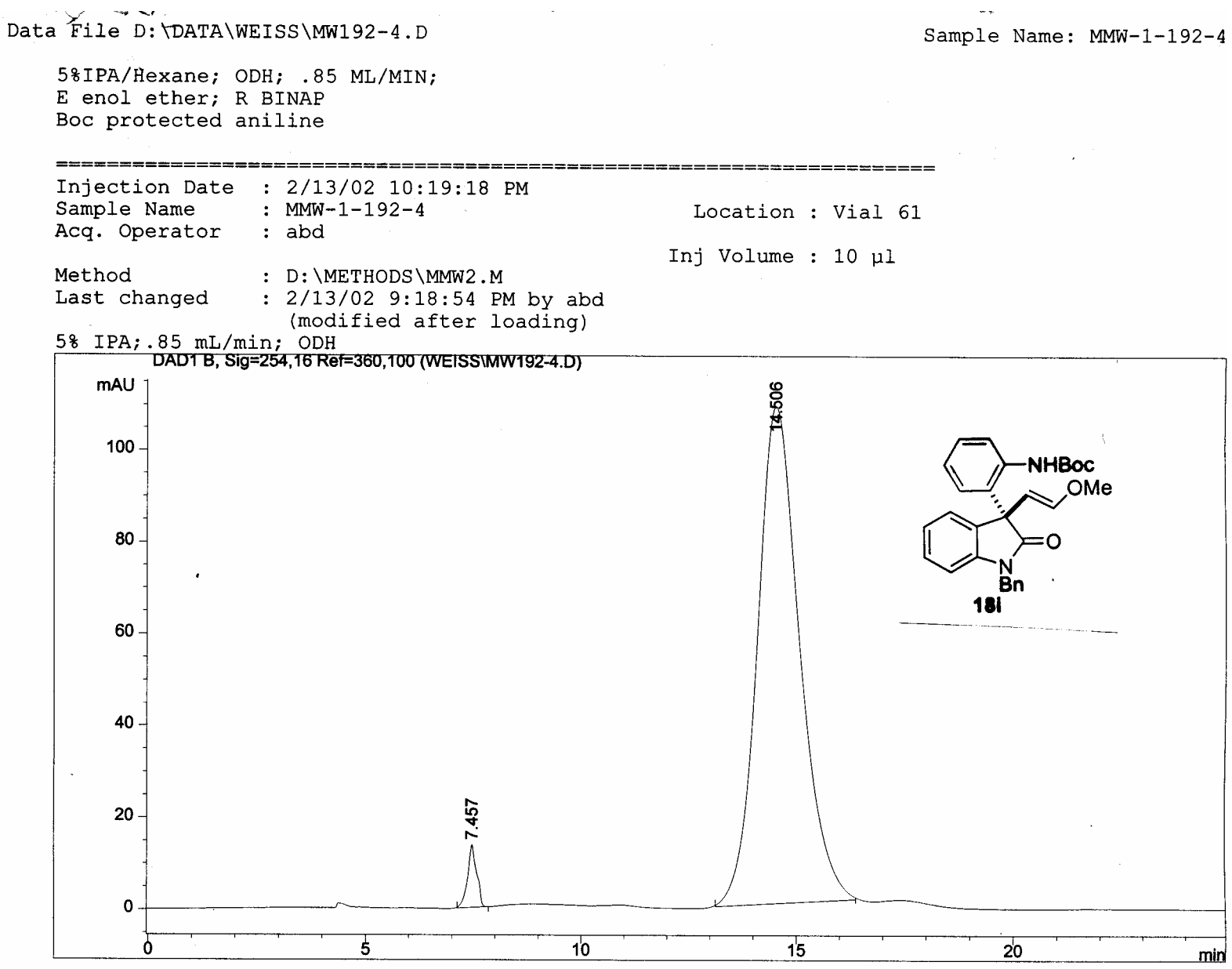

\begin{tabular}{|c|c|c|}
\hline Sorted By & : & Signa \\
\hline Multiplier & : & 1.000 \\
\hline Dilution & : & 1.000 \\
\hline
\end{tabular}

Signal 1: DAD1 B, Sig=254, 16 Ref $=360,100$

\begin{tabular}{|c|c|c|c|c|c|c|}
\hline $\begin{array}{c}\text { Peak } \\
\quad \#\end{array}$ & $\begin{array}{c}\text { RetTime } \\
\text { [min] }\end{array}$ & Type & $\begin{array}{l}\text { Width } \\
\text { [min] }\end{array}$ & $\begin{array}{c}\text { Area } \\
{\left[\mathrm{mAU}{ }^{\star} \mathrm{s}\right]}\end{array}$ & $\begin{array}{l}\text { Height } \\
\text { [mAU] }\end{array}$ & $\begin{array}{c}\text { Area } \\
\frac{9}{8}\end{array}$ \\
\hline $\begin{array}{l}1 \\
2\end{array}$ & $\begin{array}{r}7.457 \\
14.506\end{array}$ & $\begin{array}{l}\text { BP } \\
\text { BB }\end{array}$ & $\begin{array}{l}0.2006 \\
1.0230\end{array}$ & $\begin{array}{r}199.66243 \\
7369.50244\end{array}$ & $\begin{array}{r}13.65712 \\
108.41526\end{array}$ & $\begin{array}{c}2.6378 \\
97.3622\end{array}$ \\
\hline otal & : & & & 7569.16487 & 122.07238 & \\
\hline
\end{tabular}

Results obtained with enhanced integrator!

$\star \star \star$ End of Report $\star \star \star$ 


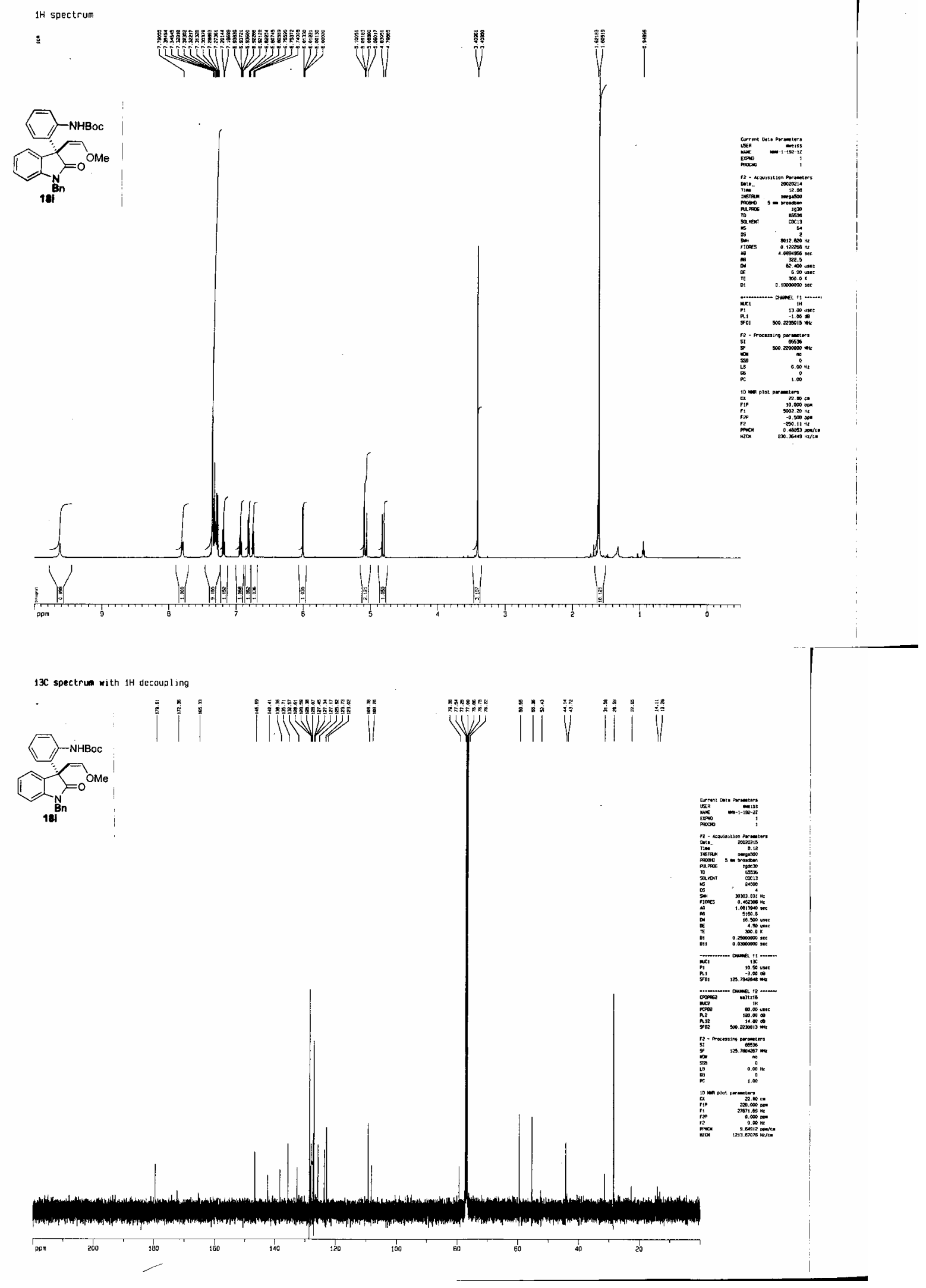


Data File D: \DATA \WEISS \MW192-3.D
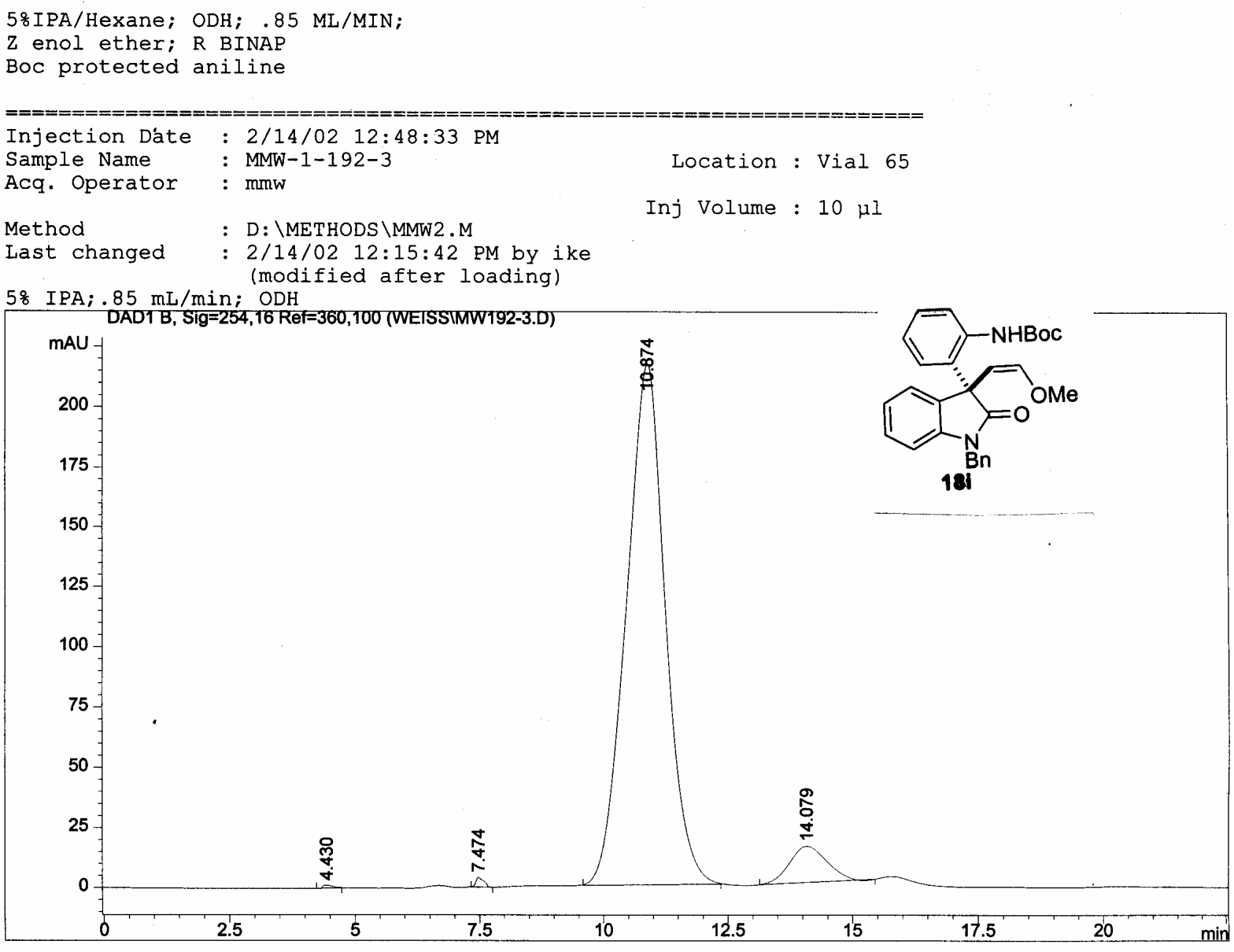

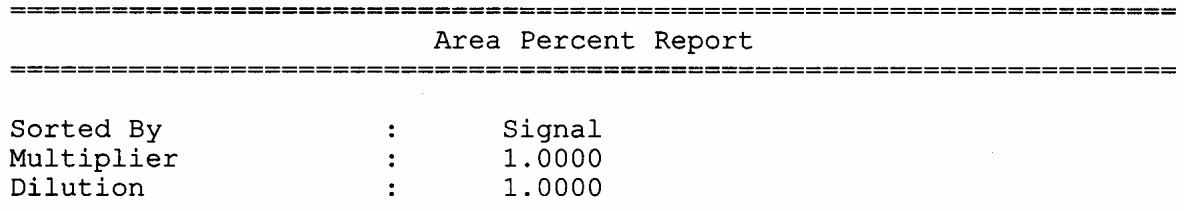

Signal 1: DAD1 B, Sig=254,16 Ref=360,100

\begin{tabular}{|c|c|c|c|c|c|c|}
\hline $\begin{array}{c}\text { Peak } \\
\#\end{array}$ & $\begin{array}{l}\text { RetTime } \\
\text { [min] }\end{array}$ & Type & $\begin{array}{l}\text { Width } \\
\text { [min] }\end{array}$ & $\begin{array}{c}\text { Area } \\
{\left[\mathrm{mAU} U^{\star} \mathrm{s}\right]}\end{array}$ & $\begin{array}{l}\text { Height } \\
\text { [mAU] }\end{array}$ & $\begin{array}{c}\text { Area } \\
\frac{\circ}{8}\end{array}$ \\
\hline $\begin{array}{l}1 \\
2 \\
3 \\
4\end{array}$ & $\begin{array}{r}4.430 \\
7.474 \\
10.874 \\
14.079\end{array}$ & $\begin{array}{l}\mathrm{PB} \\
\mathrm{BP} \\
\mathrm{BB} \\
\mathrm{BP}\end{array}$ & $\begin{array}{l}0.1951 \\
0.1499 \\
0.8054 \\
0.7796\end{array}$ & $\begin{array}{r}16.56817 \\
46.66462 \\
1.18401 \mathrm{e} 4 \\
781.31079\end{array}$ & $\begin{array}{r}1.17046 \\
4.25524 \\
216.71307 \\
15.15219\end{array}$ & $\begin{array}{r}0.1306 \\
0.3679 \\
93.3420 \\
6.1595\end{array}$ \\
\hline Total & Ls : & & & $1.26846 \mathrm{e} 4$ & 237.29095 & \\
\hline
\end{tabular}




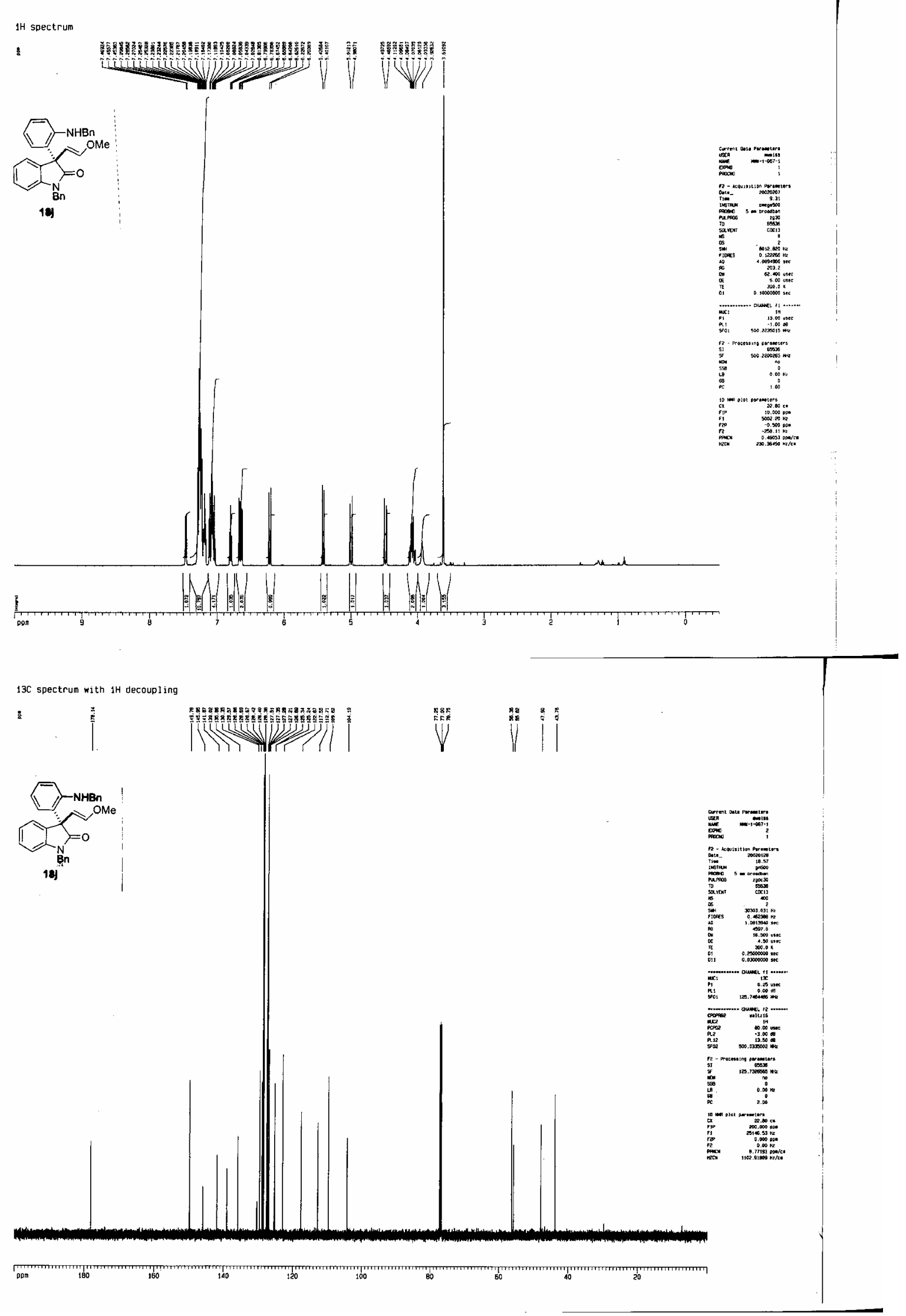


Data File D: \DATA \MWEISS \MW186-6C.D

108IPA/HX; ODH: $.85 \mathrm{~mL} / \mathrm{min}$

$E$ enol ether; R BINAP

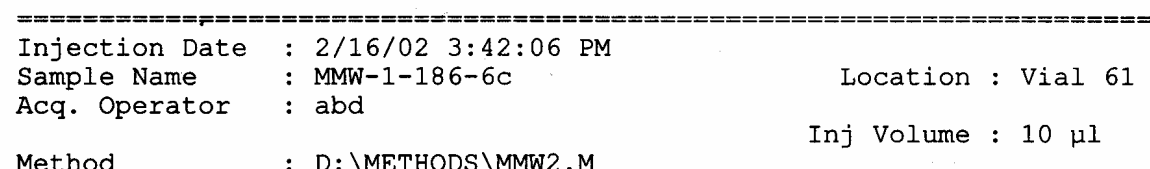

Last changed : 2/16/02 3:36:04 PM by abd (modified after loading)

$10 \% \mathrm{IPA} ; .85 \mathrm{~mL} / \mathrm{min} ; \mathrm{ODH}$

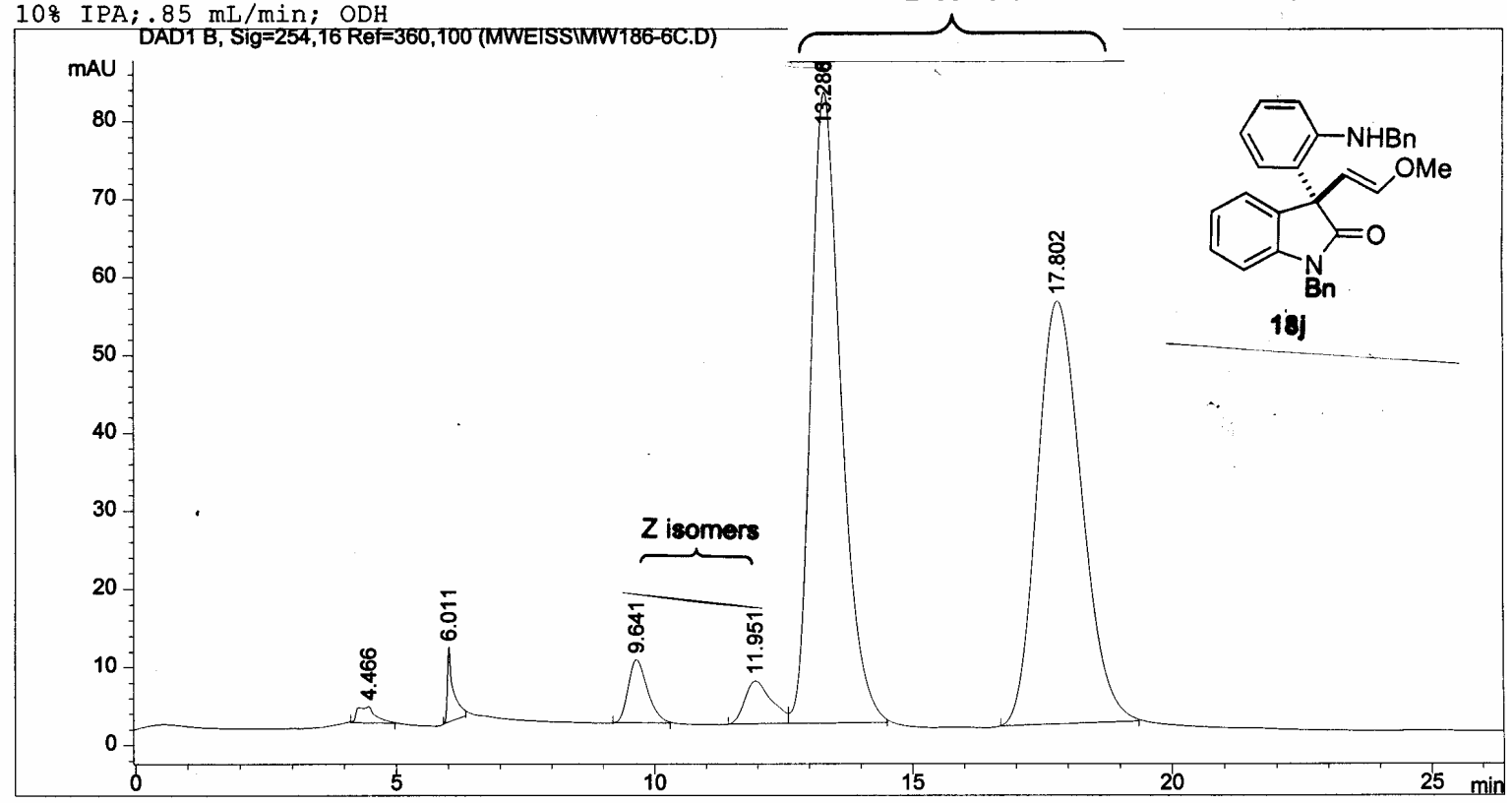

\begin{tabular}{|c|c|c|}
\hline \multicolumn{3}{|c|}{ Area Perce } \\
\hline Sorted By & $:$ & Sig: \\
\hline Multiplier & : & 1.000 \\
\hline Dilution & : & 1.0000 \\
\hline
\end{tabular}

Signal 1: DAD1 B, Sig=254,16 Ref=360,100

\begin{tabular}{|c|c|c|c|c|c|c|}
\hline $\begin{array}{c}\text { Peak } \\
\#\end{array}$ & $\begin{array}{c}\text { RetTime } \\
\text { [min] }\end{array}$ & Type & $\begin{array}{c}\text { Width } \\
\text { [min] }\end{array}$ & $\begin{array}{c}\text { Area } \\
{\left[\mathrm{mAU}^{\star} \mathrm{s}\right]}\end{array}$ & $\begin{array}{l}\text { Height } \\
\text { [mAU] }\end{array}$ & $\begin{array}{c}\text { Area } \\
\frac{8}{8}\end{array}$ \\
\hline $\begin{array}{l}-1 \\
2 \\
3 \\
4 \\
5 \\
6\end{array}$ & $\begin{array}{r}4.466 \\
6.011 \\
9.641 \\
11.951 \\
13.286 \\
17.802\end{array}$ & $\begin{array}{l}\text { BB } \\
\text { PB } \\
\text { PB } \\
\text { BV } \\
\text { VB } \\
\text { BB }\end{array}$ & $\begin{array}{l}0.3020 \\
0.1070 \\
0.3958 \\
0.5186 \\
0.6224 \\
0.9237\end{array}$ & $\begin{array}{r}51.00165 \\
76.92011 \\
212.45720 \\
200.32639 \\
3292.91650 \\
3230.47925\end{array}$ & $\begin{array}{r}2.10998 \\
9.65169 \\
8.15966 \\
5.53038 \\
80.98792 \\
54.33356\end{array}$ & $\begin{array}{r}0.7220 \\
1.0889 \\
3.0076 \\
2.8358 \\
46.6148 \\
45.7309\end{array}$ \\
\hline Total & Is : & & & 7064.10110 & 160.77318 & \\
\hline
\end{tabular}


Supporting Information January 28, 2003 Dounay, Hatanaka, Kodanko, Oestreich, Overman*, Pfeifer and Weiss

$\mathrm{Kh}-\mathrm{I}-80-0911$
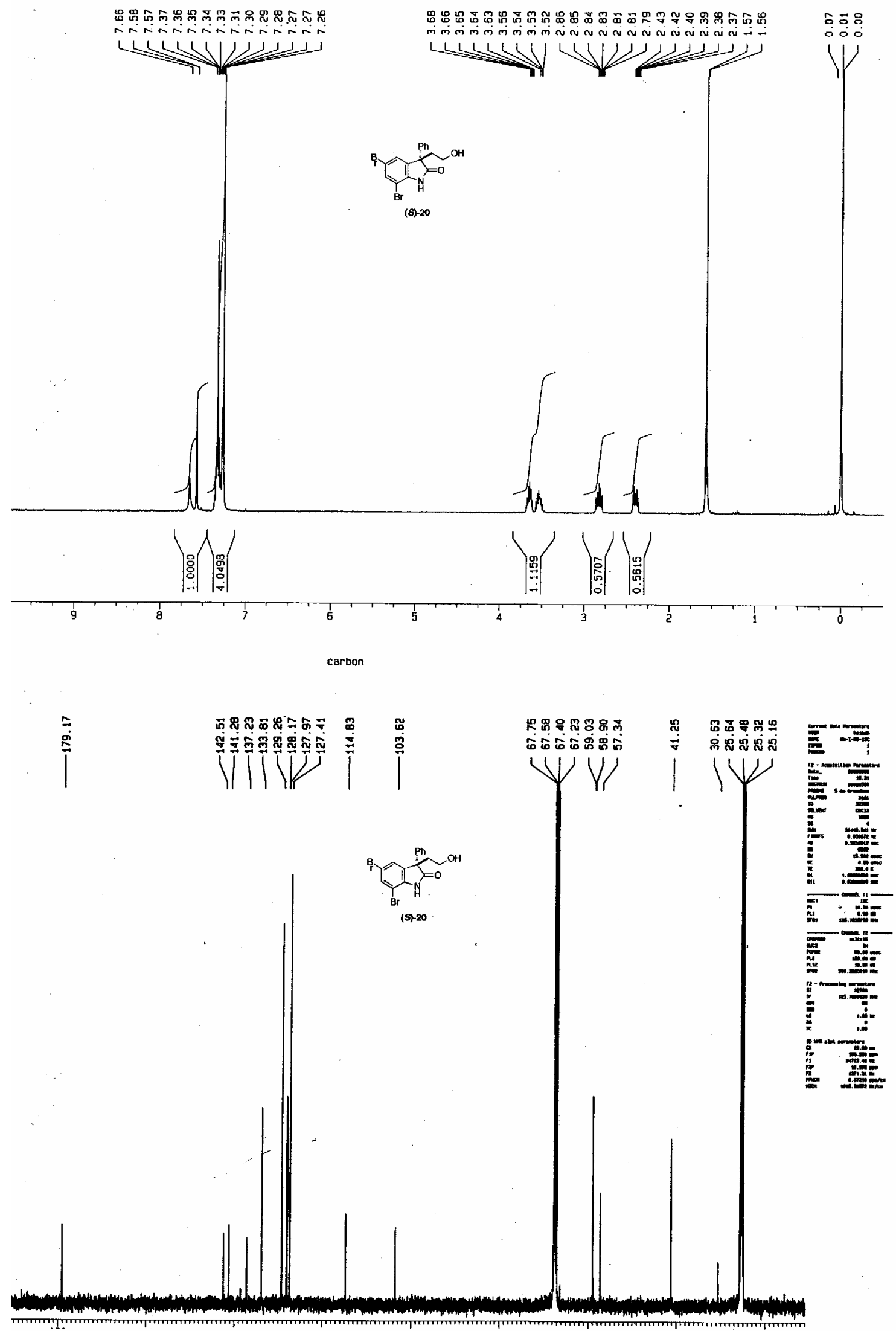


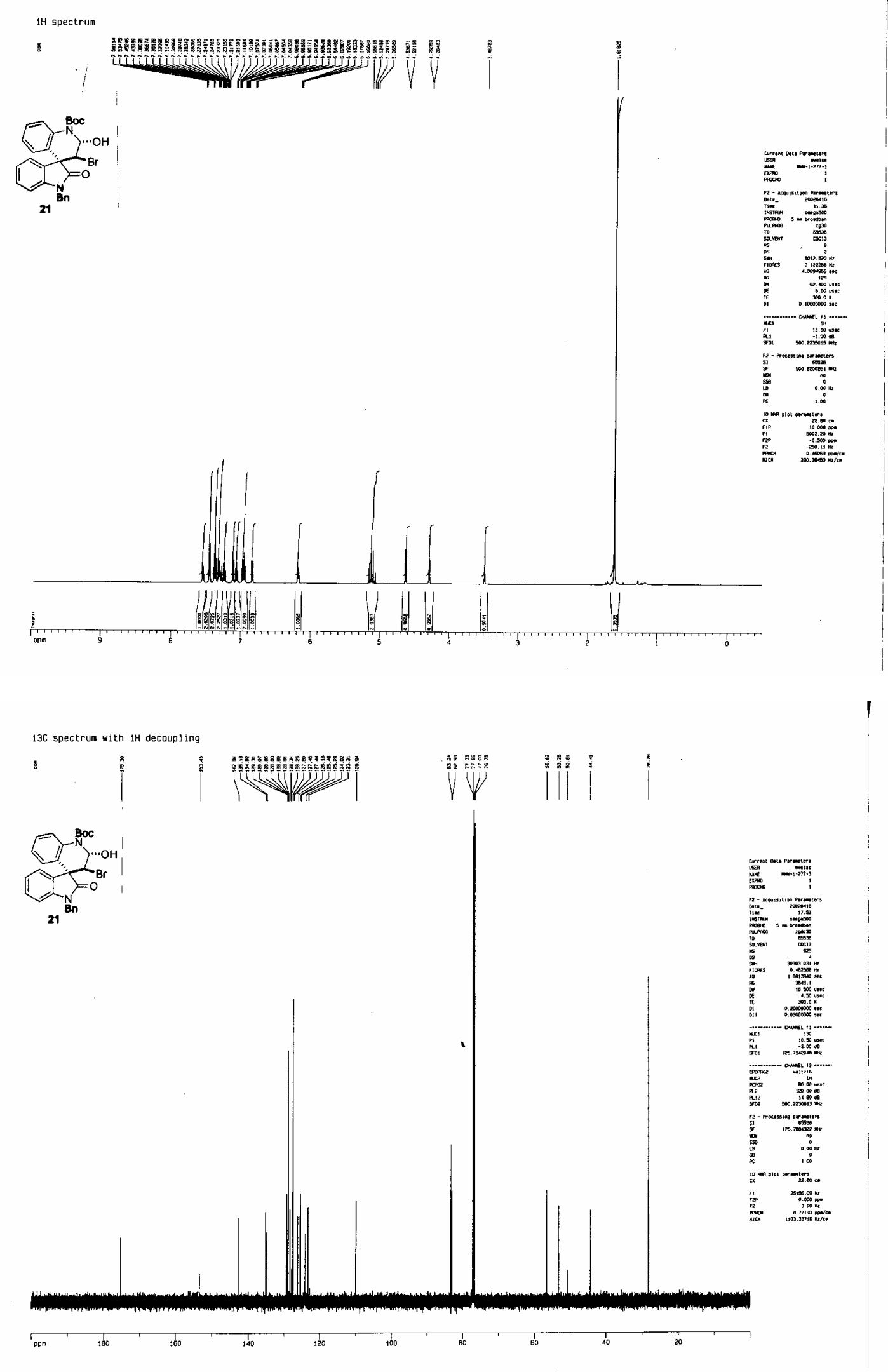



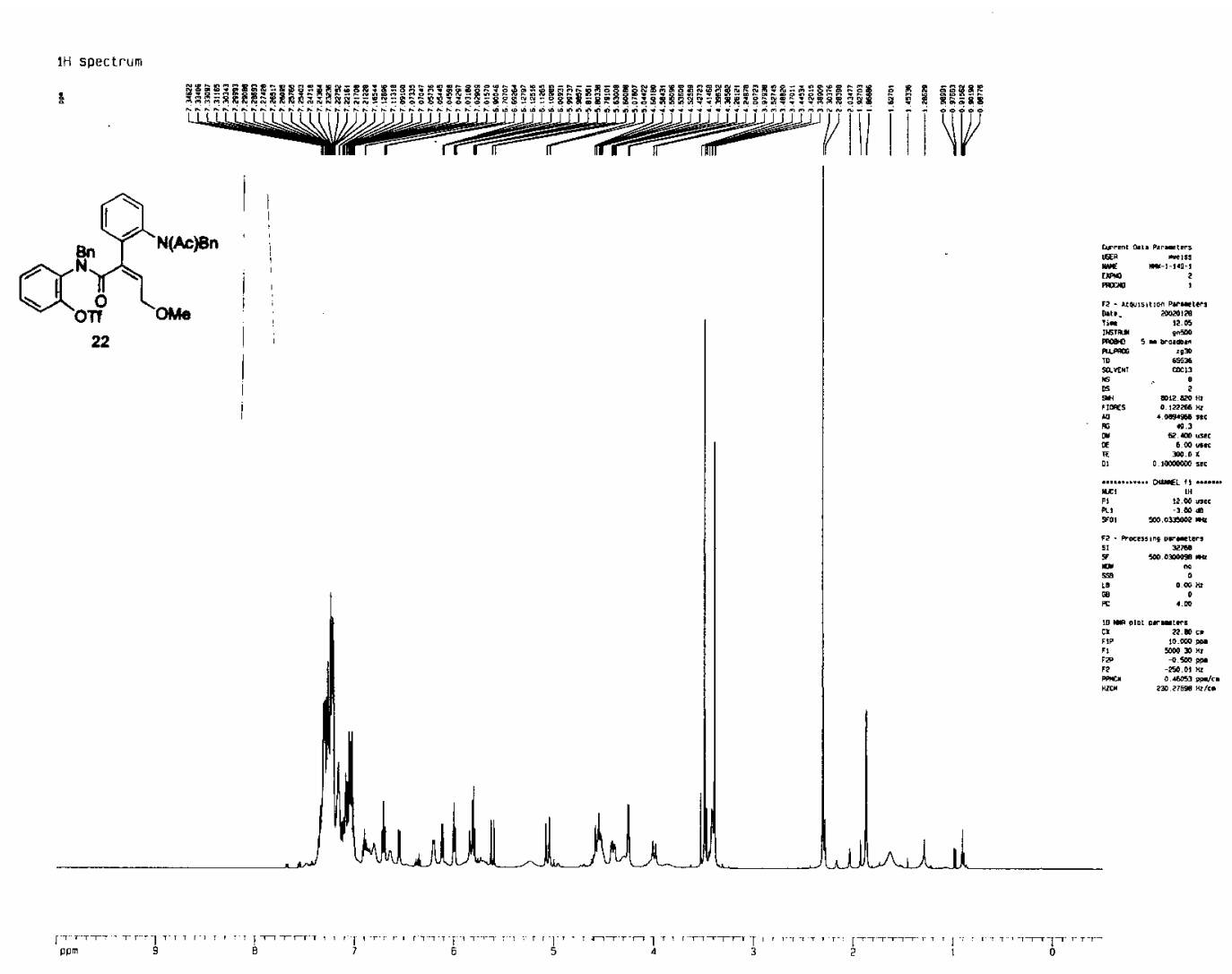

13 C spectrum with $1 \mathrm{H}$ decoupling

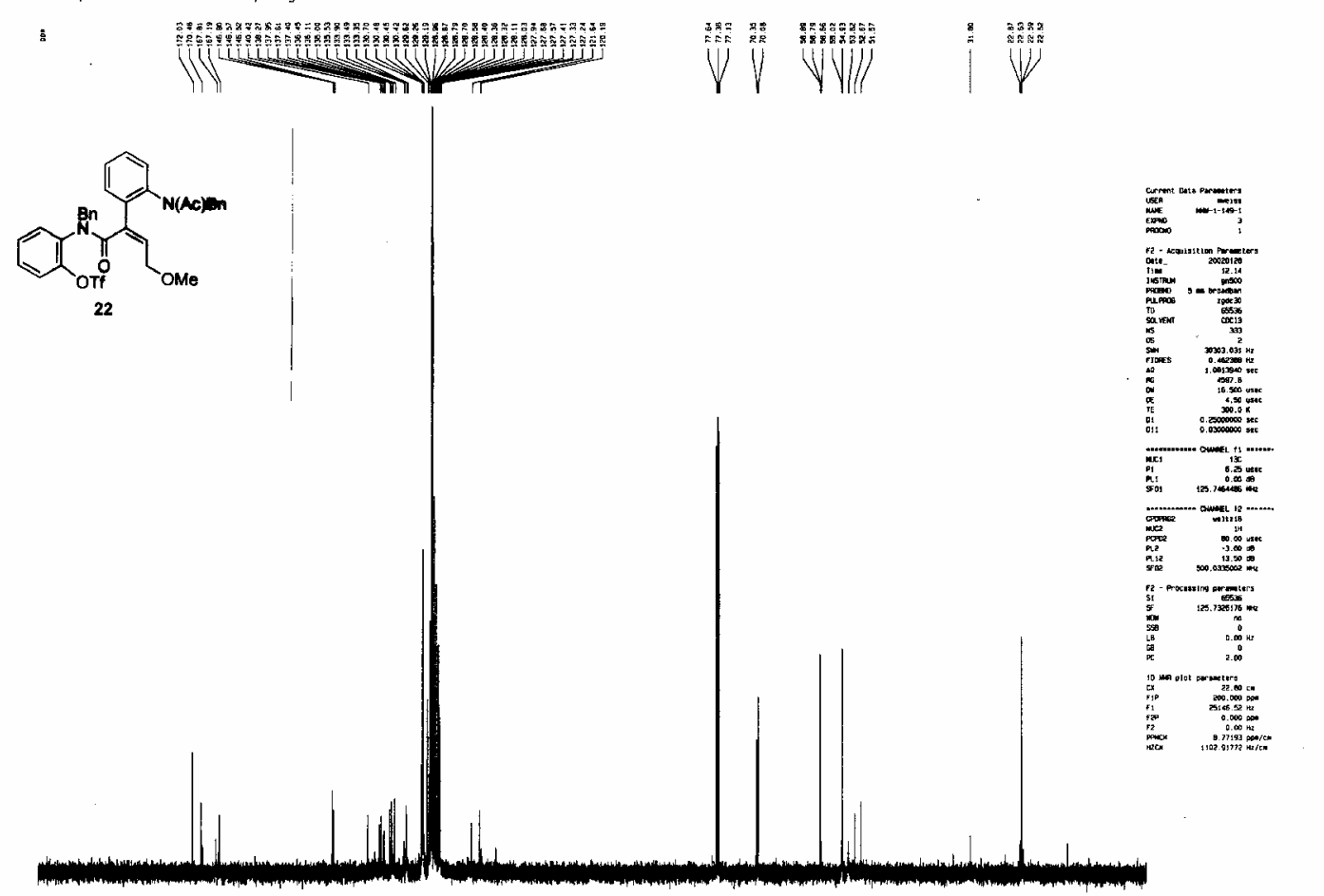

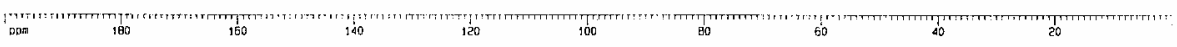


Supporting Information January 28, 2003 Dounay, Hatanaka, Kodanko, Oestreich, Overman*, Pfeifer and Weiss

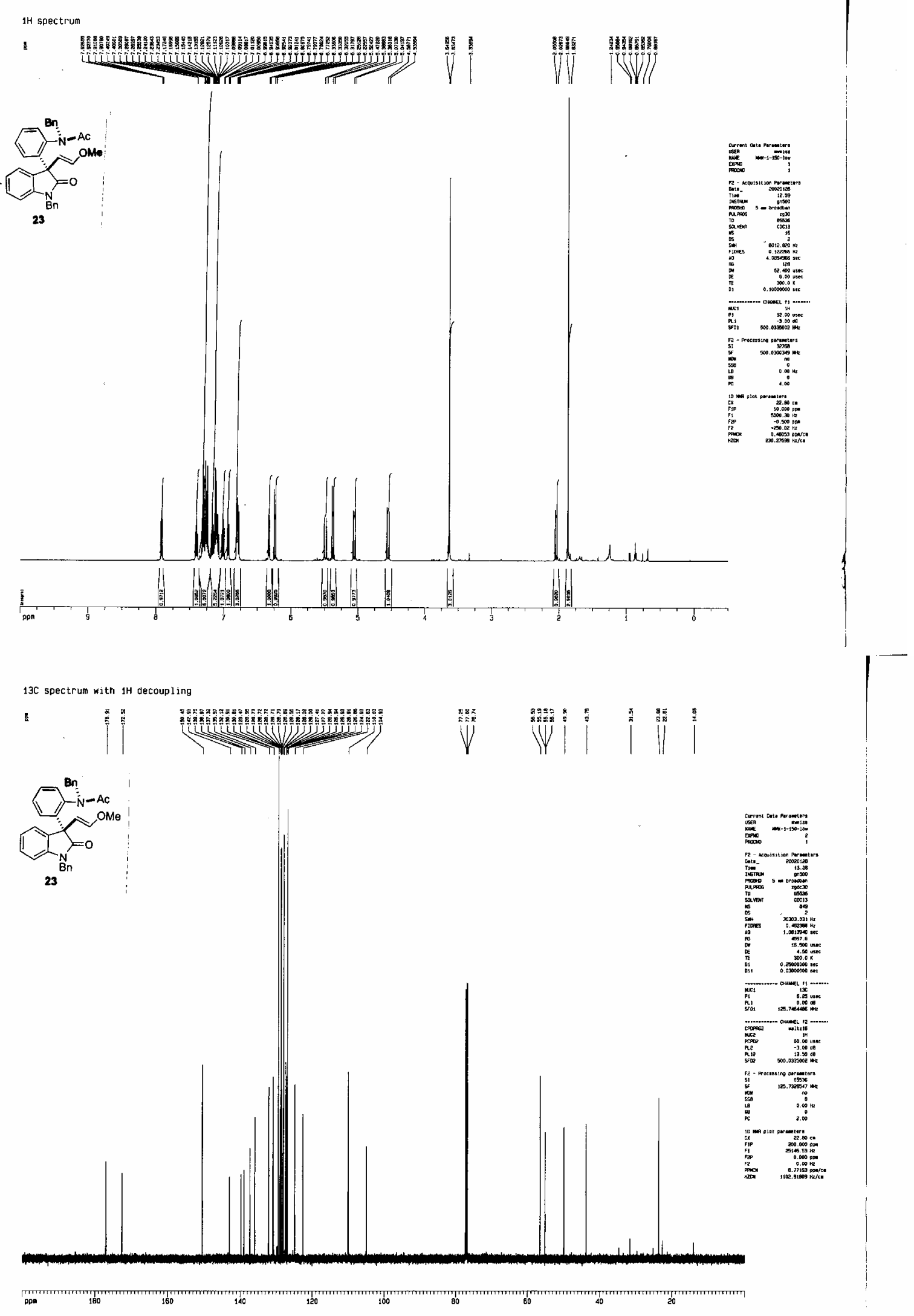


Data File D: \DATA \MWEISS $\backslash M W 147-2$. D

$108 \mathrm{IPA} / \mathrm{hex} ; \mathrm{y}$-BINAP; $.85 \mathrm{~mL} / \mathrm{min}$ low Rf; ODH

\begin{tabular}{|c|c|}
\hline $\begin{array}{l}\text { Injection Date } \\
\text { Sample Name } \\
\text { Acq. Operator }\end{array}$ & $\begin{array}{l}: 1 / 9 / 0212: 04: 25 \mathrm{PM} \\
: \text { MMW-1-147-2 } \\
: \text { abd }\end{array}$ \\
\hline $\begin{array}{l}\text { Method } \\
\text { Last changed }\end{array}$ & $\begin{array}{l}: \text { D: \METHODS } \backslash \text { WEISS.M } \\
: 1 / 9 / 02 \text { 11:28:30 AM by abd } \\
\text { (modified after loading) }\end{array}$ \\
\hline
\end{tabular}

108 IPA-Hexanes

$.85 \mathrm{~mL} / \mathrm{min}$;

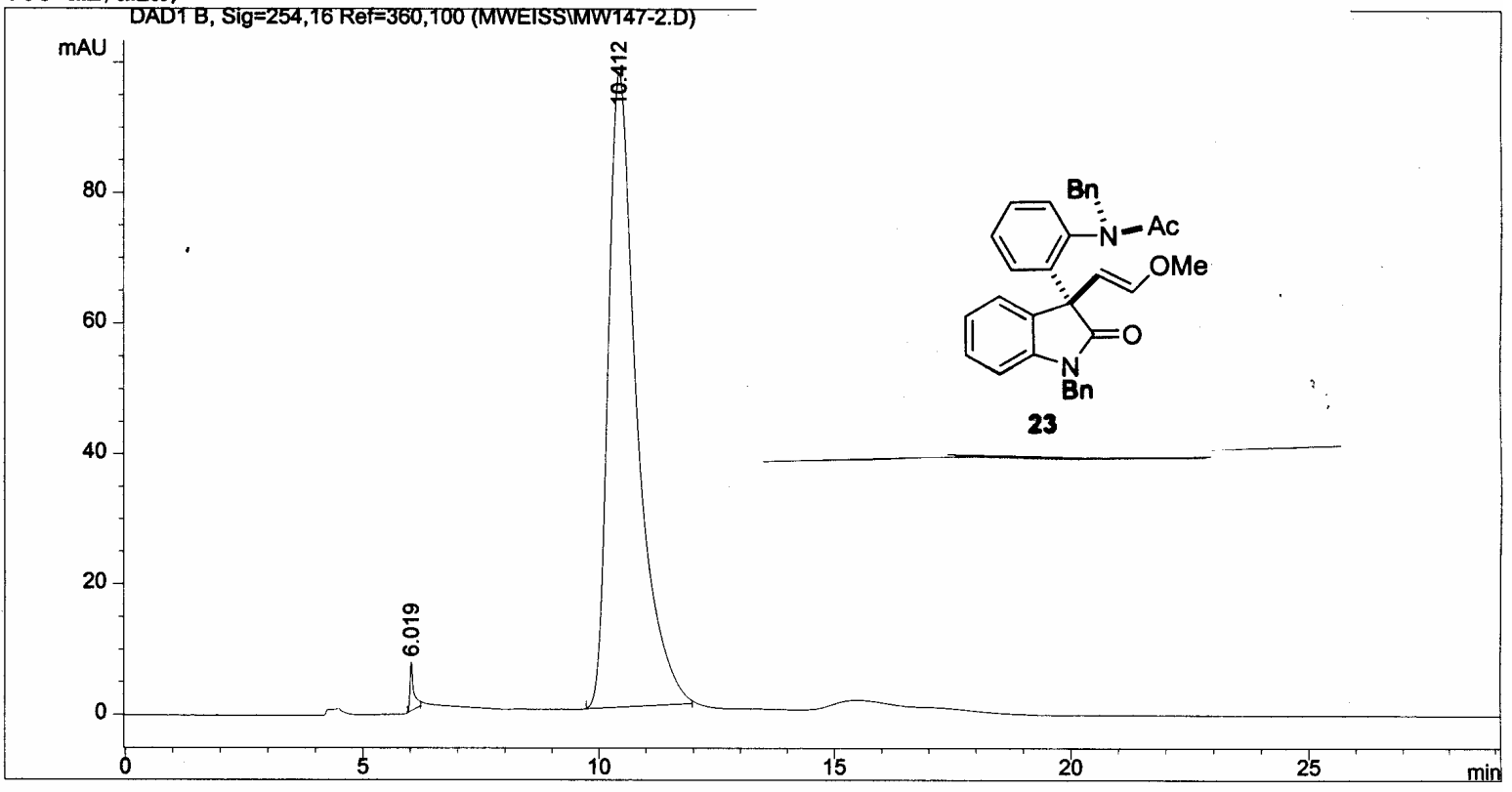

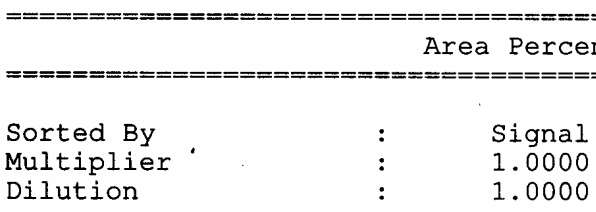

Signal 1: DAD1 B, Sig=254,16 Ref=360,100

\begin{tabular}{|c|c|c|c|c|c|c|}
\hline $\begin{array}{c}\text { Peak } \\
\#\end{array}$ & $\begin{array}{c}\text { RetTime } \\
{[\text { min] }}\end{array}$ & Type & $\begin{array}{l}\text { Width } \\
\text { [min] }\end{array}$ & $\begin{array}{c}\text { Area } \\
{\left[\mathrm{mAU}^{*} \mathrm{~s}\right]}\end{array}$ & $\begin{array}{l}\text { Height } \\
\text { [mAU] }\end{array}$ & $\begin{array}{c}\text { Area } \\
\frac{\circ}{\partial}\end{array}$ \\
\hline $\begin{array}{l}1 \\
2\end{array}$ & $\begin{array}{r}6.019 \\
10.412\end{array}$ & $\begin{array}{l}\text { PB } \\
\text { BB }\end{array}$ & $\begin{array}{l}0.0778 \\
0.6289\end{array}$ & $\begin{array}{r}40 . \\
4110 .\end{array}$ & $\begin{array}{r}7.44352 \\
97.29494\end{array}$ & $\begin{array}{r}0.9805 \\
99.0195\end{array}$ \\
\hline & & & & 4150.89439 & 104.73846 & \\
\hline
\end{tabular}

Results obtained with enhanced integrator!

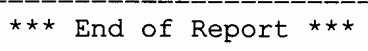




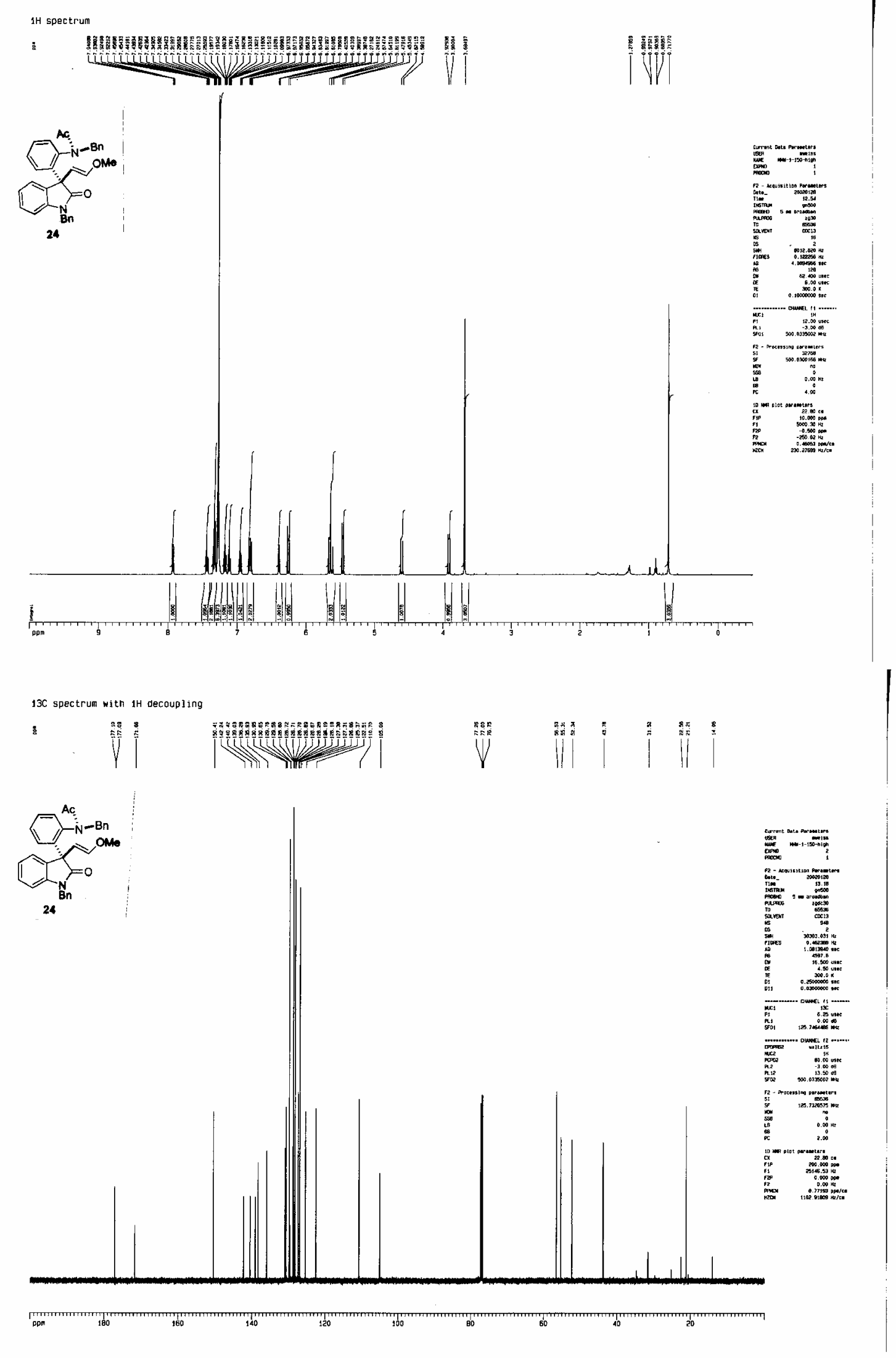




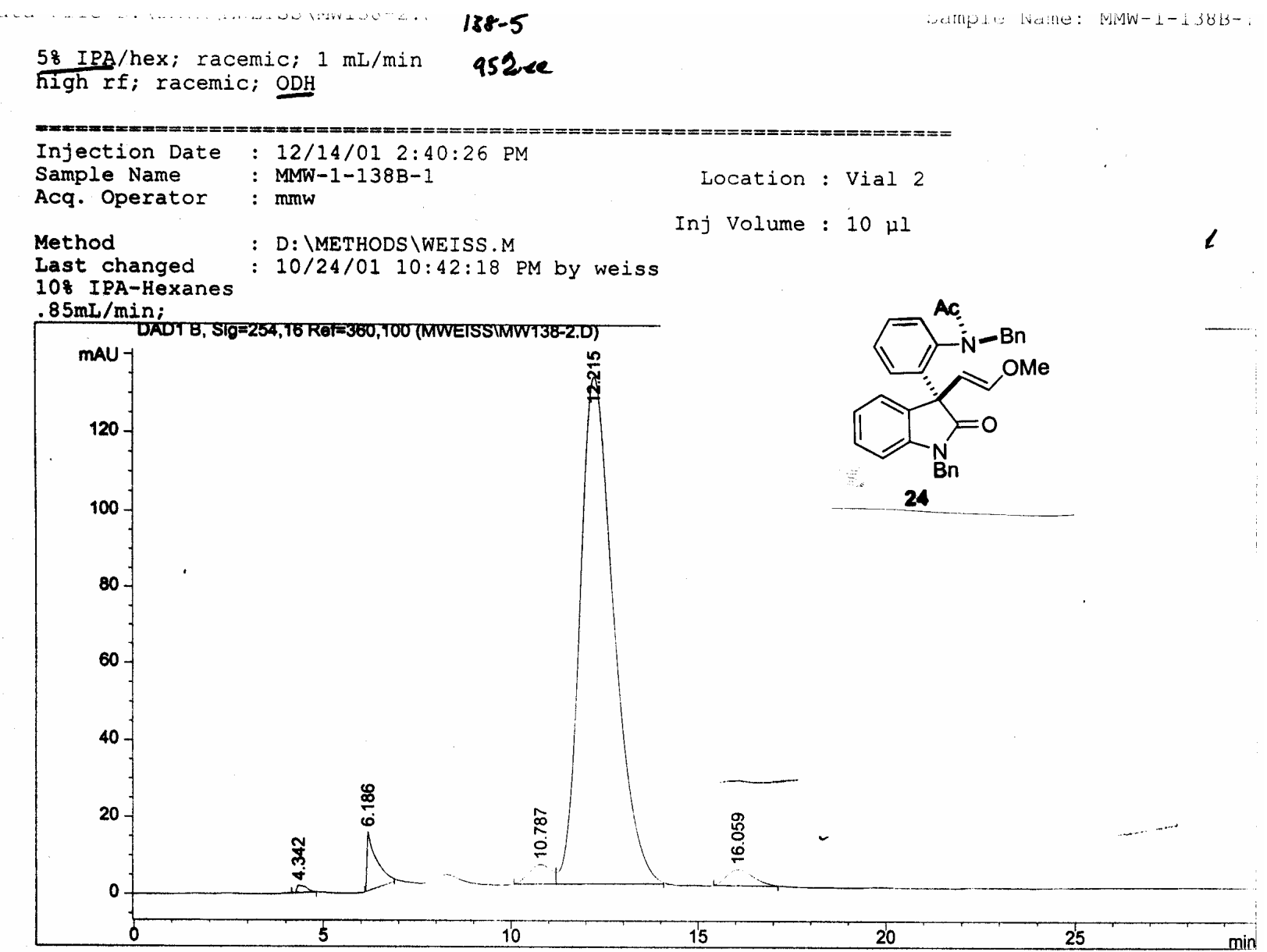

Area Percent Report

$\begin{array}{lll} & & \\ \text { Sorted By } & : & \text { Signal } \\ \text { Multiplier } & : & 1.0000 \\ \text { Dilution } & : & 1.0000\end{array}$

Signal 1: DADI B, Sig=254,16 Ref $=360,100$

\begin{tabular}{|c|c|c|c|c|c|c|}
\hline $\begin{array}{c}\text { Peak } \\
\#\end{array}$ & $\begin{array}{l}\text { RetTime } \\
\text { [min] }\end{array}$ & Type & $\begin{array}{l}\text { Width } \\
\text { [min] }\end{array}$ & $\begin{array}{c}\text { Area } \\
{\left[\mathrm{mAU}^{\star} \mathrm{s}\right]}\end{array}$ & $\begin{array}{l}\text { Height } \\
\text { [MAU] }\end{array}$ & $\begin{array}{c}\text { Area } \\
8\end{array}$ \\
\hline $\begin{array}{l}1 \\
2 \\
3 \\
4 \\
5\end{array}$ & $\begin{array}{r}4.342 \\
6.186 \\
10.787 \\
12.215 \\
16.059\end{array}$ & $\begin{array}{l}\text { BB } \\
\text { PB } \\
\text { BV } \\
\text { VB } \\
\text { BB }\end{array}$ & $\begin{array}{l}0.2399 \\
0.2196 \\
0.5927 \\
0.9573 \\
0.6929\end{array}$ & $\begin{array}{r}33.94003 \\
271.95898 \\
210.47023 \\
8297.23145 \\
207.17273\end{array}$ & $\begin{array}{r}1.86829 \\
15.43544 \\
5.08749 \\
131.64890 \\
4.17521\end{array}$ & $\begin{array}{r}0.3762 \\
3.0148 \\
2.3332 \\
91.9792 \\
2.2966\end{array}$ \\
\hline Tot & : & & & 9020.77342 & 158.21531 & \\
\hline
\end{tabular}

Results obtained with enhanced integrator!

$\star \star \star$ End of Report $* \star *$

Chiral 12/14/01 3:09:45 PM mmw

Page 1 of 1 4 

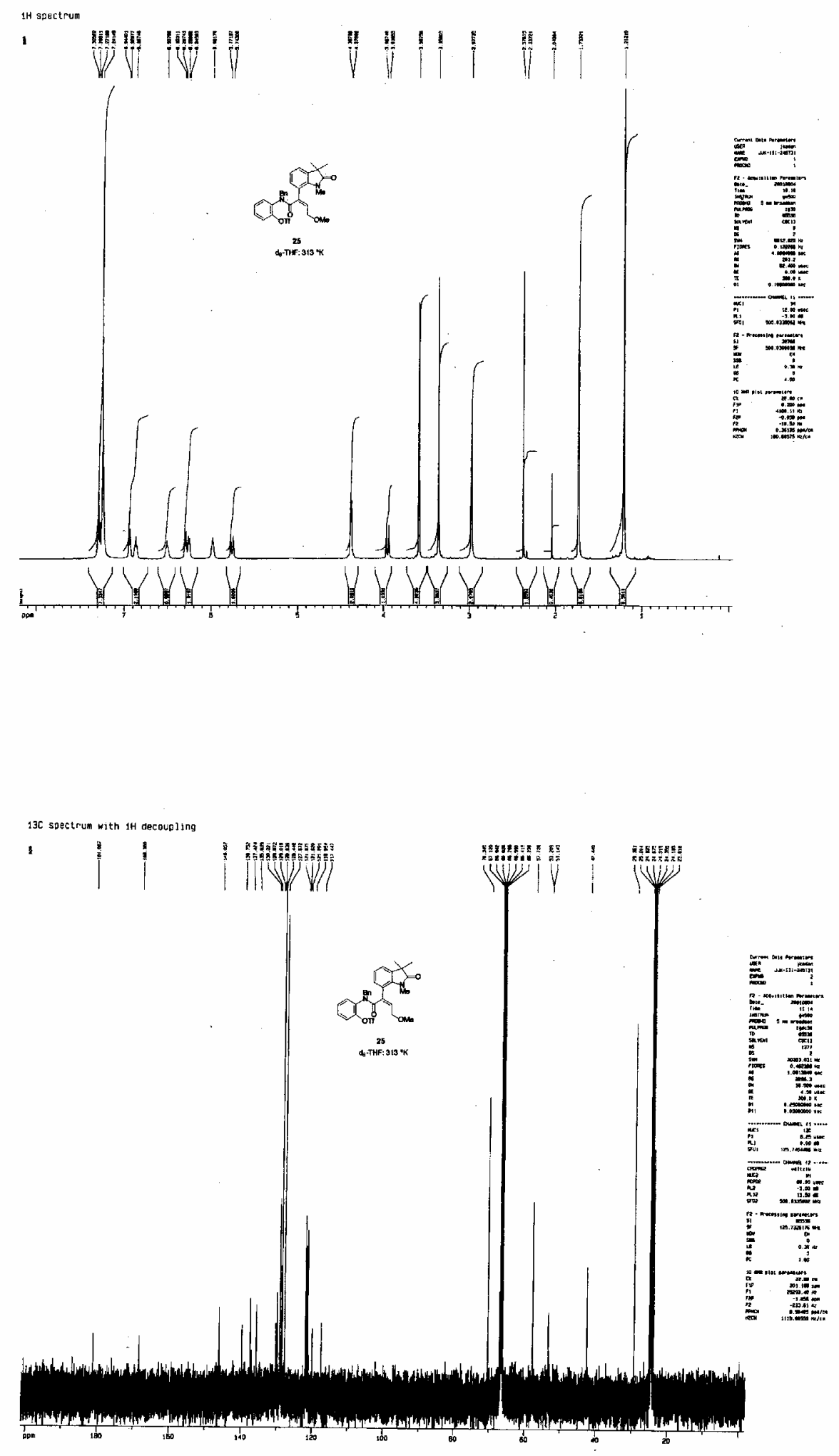


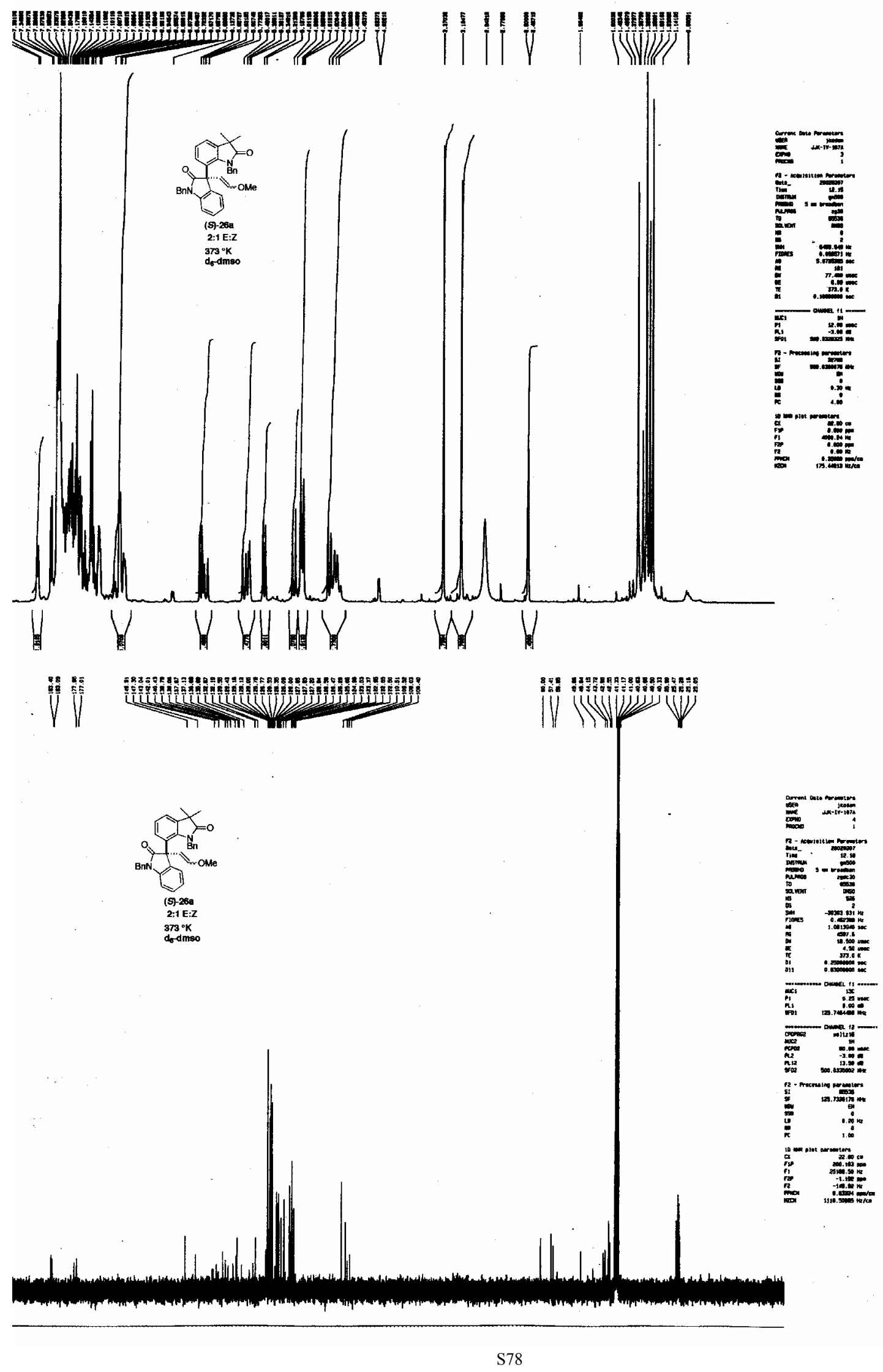


Supporting Information January 28, 2003 Dounay, Hatanaka, Kodanko, Oestreich, Overman*, Pfeifer and Weiss

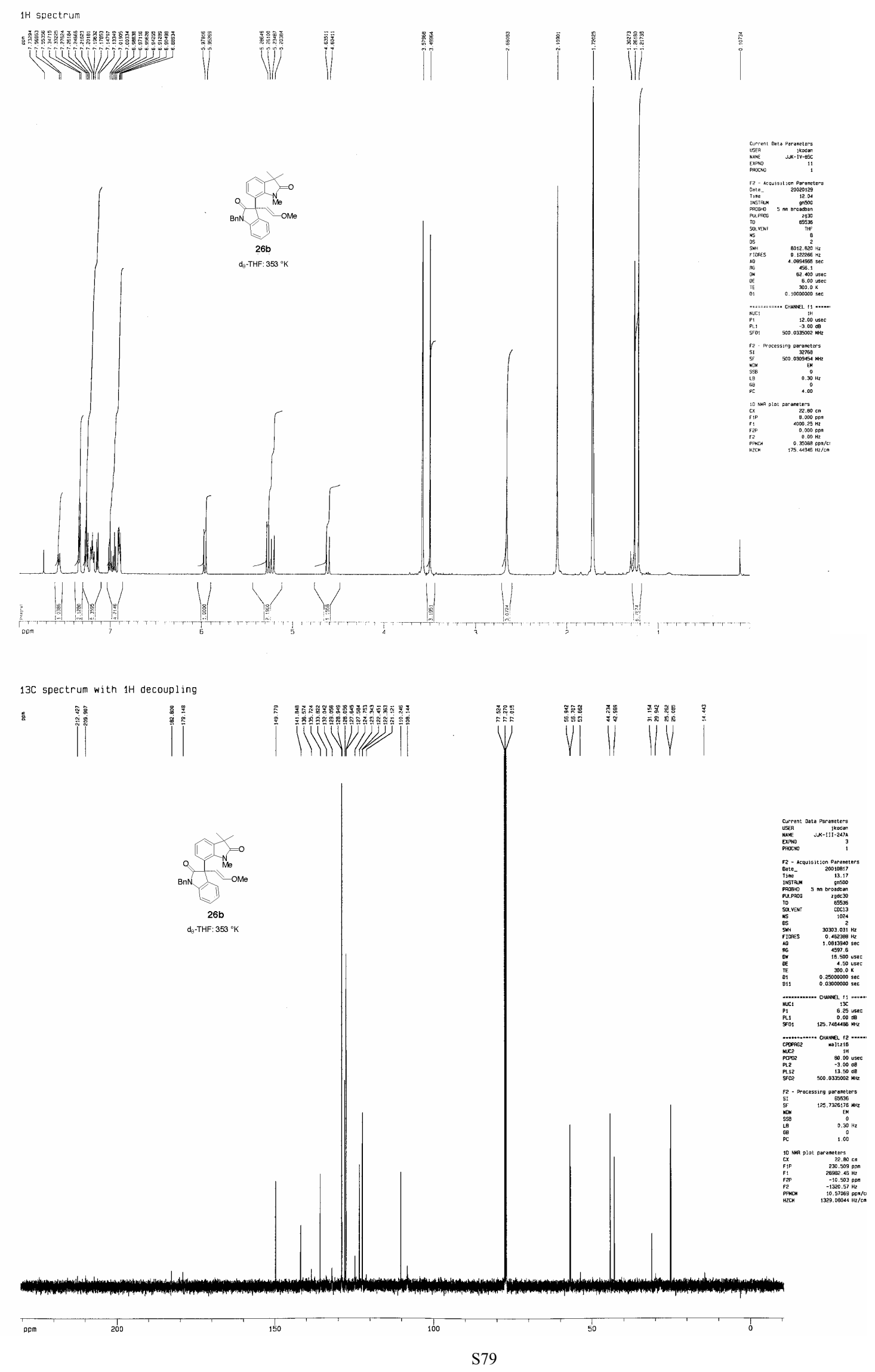



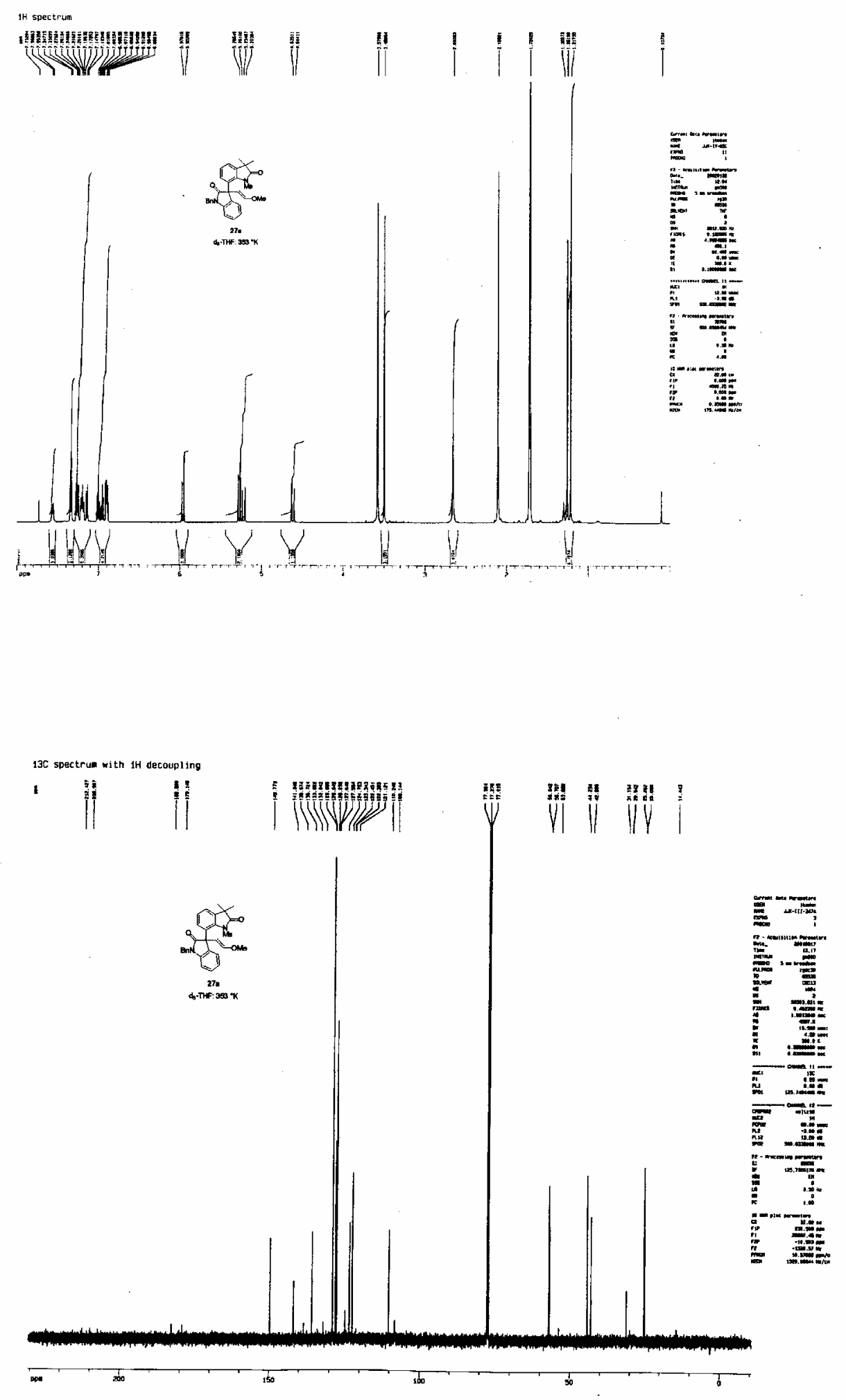
$y$
Proton
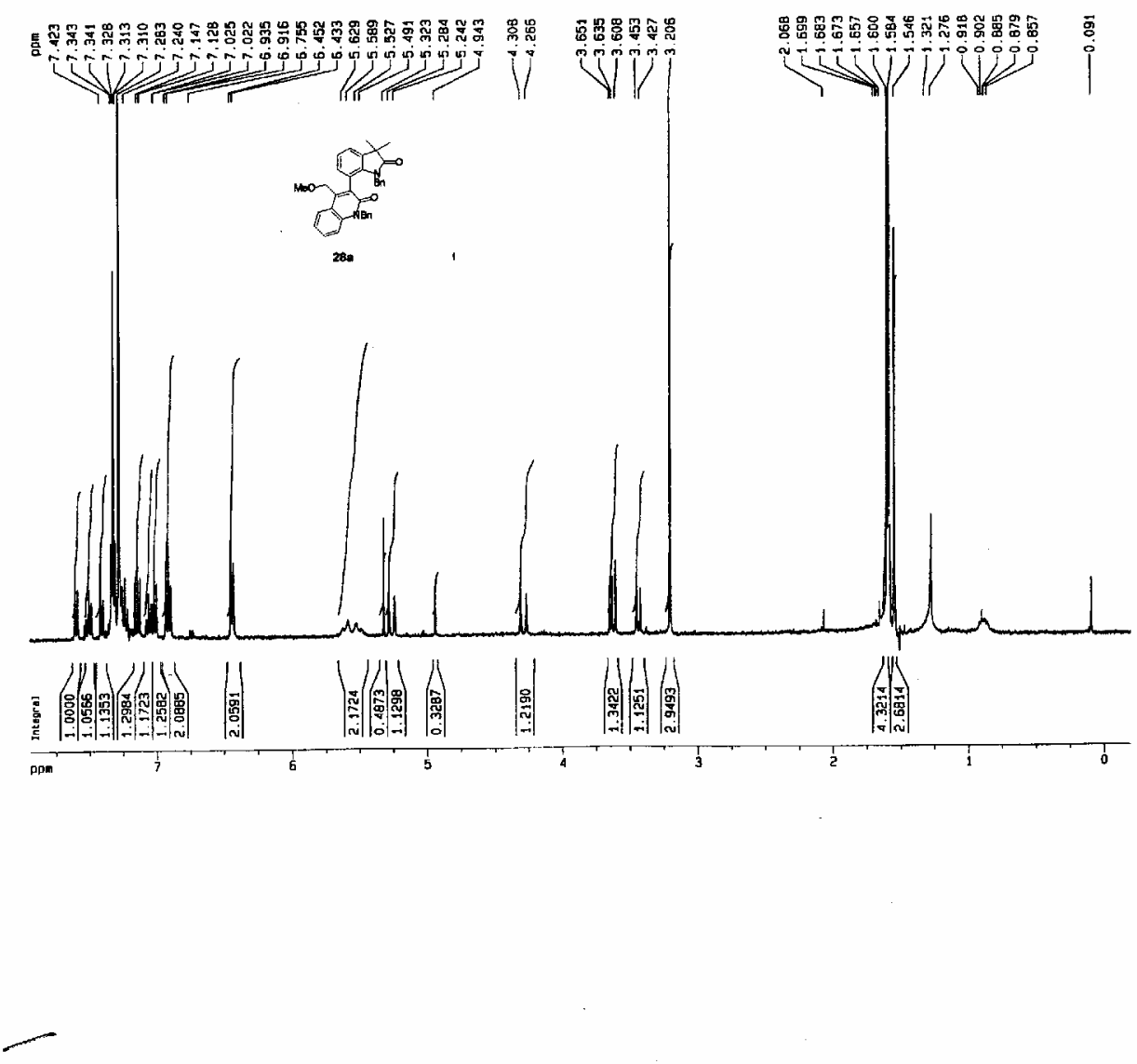

13C seetrum with 1H decoupling

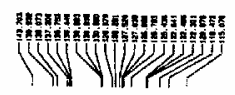

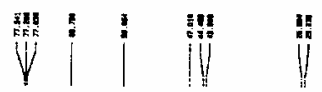
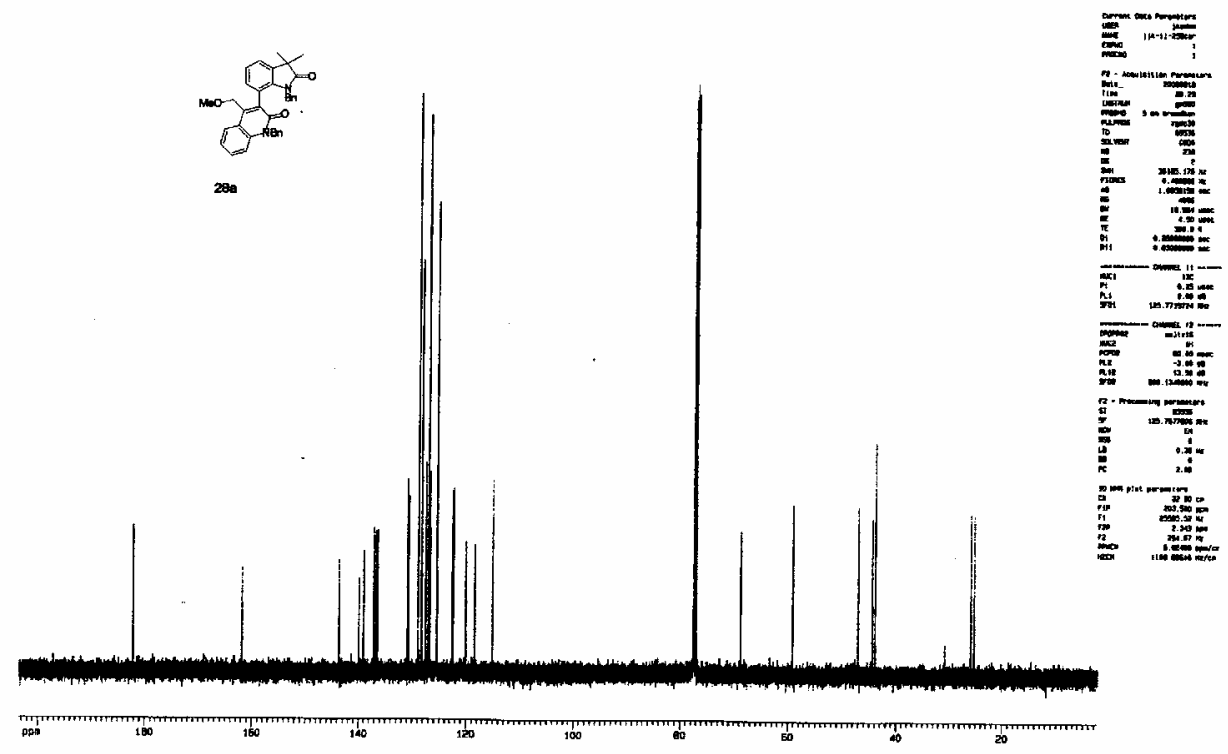

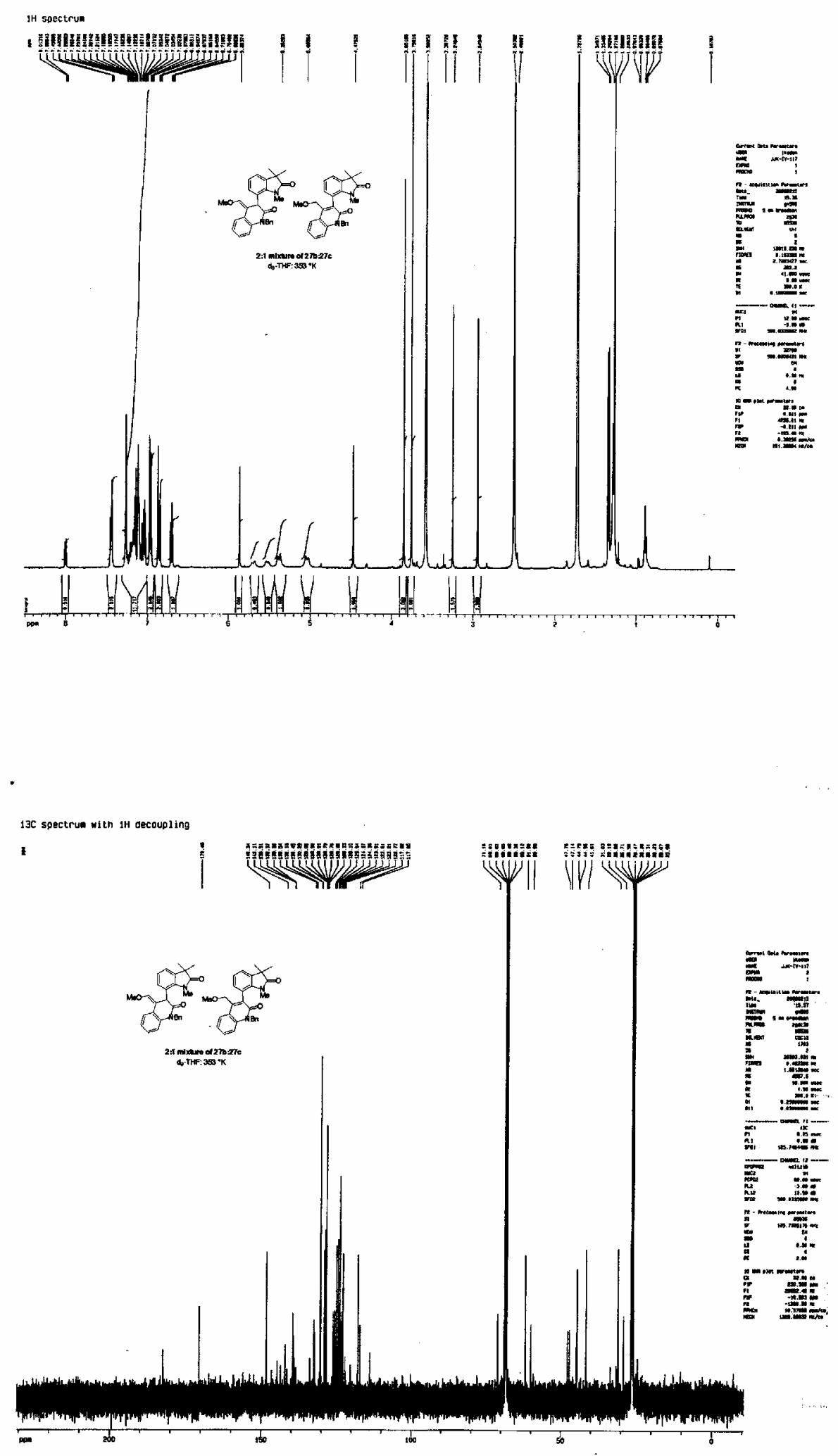

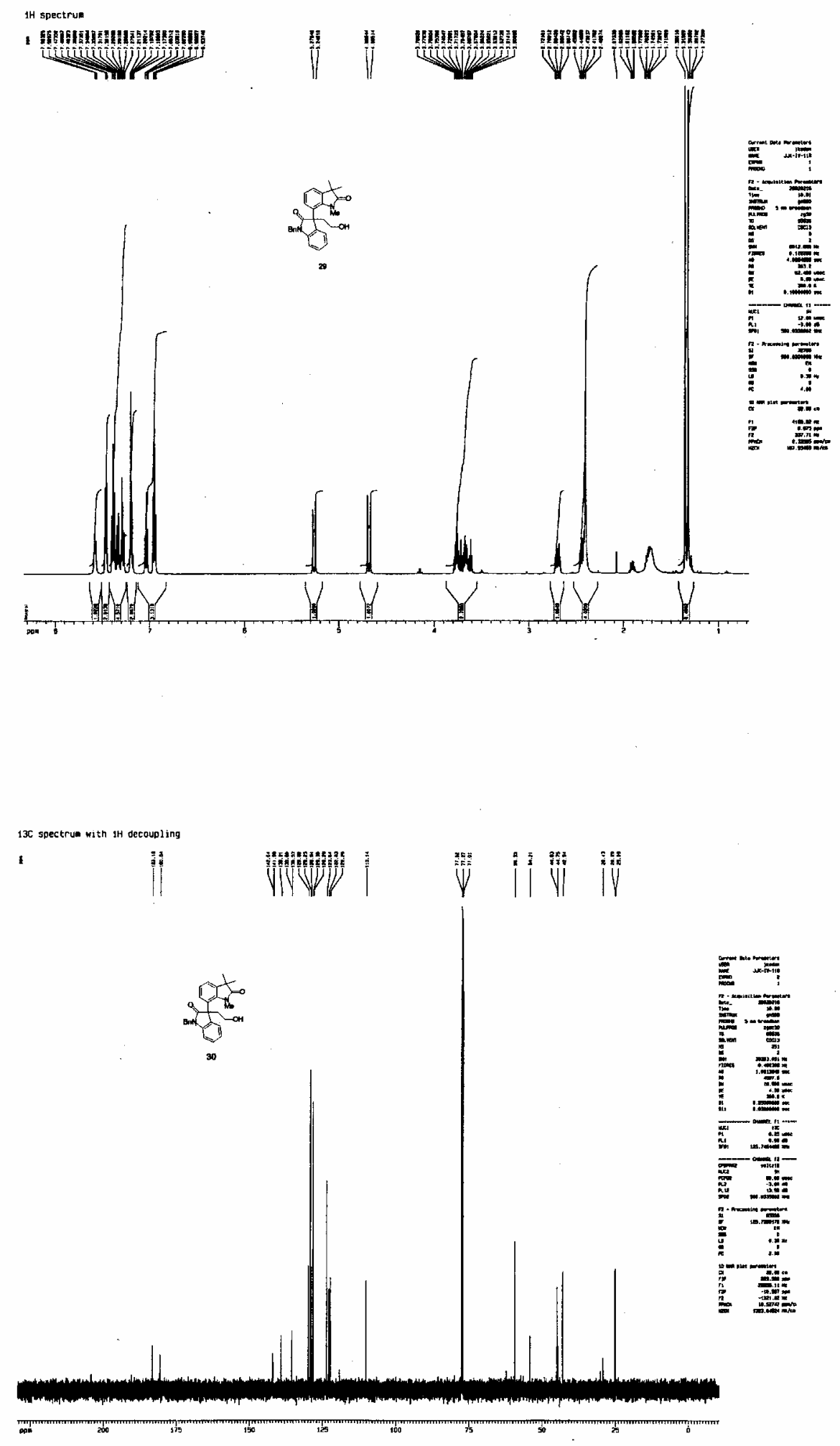


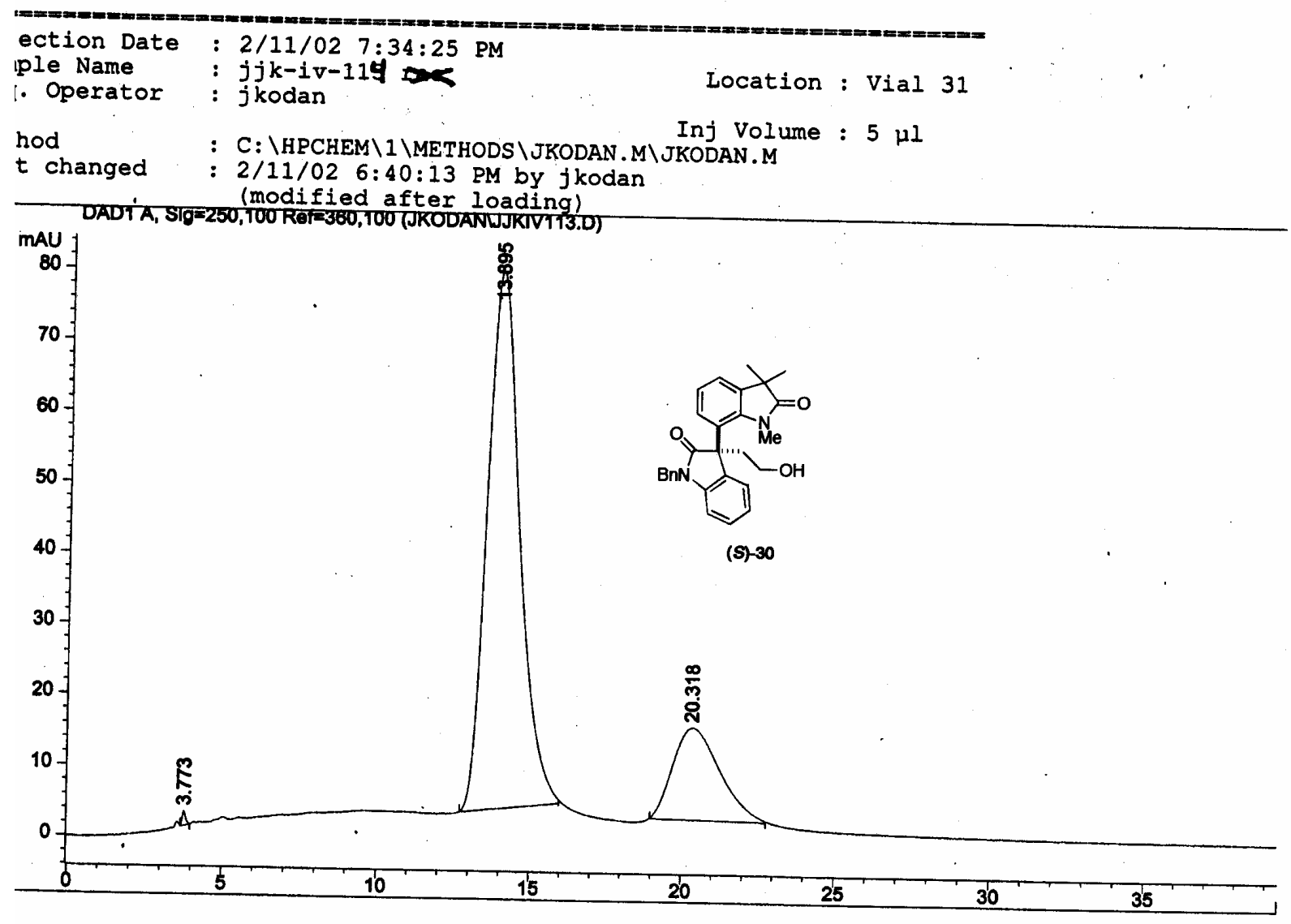

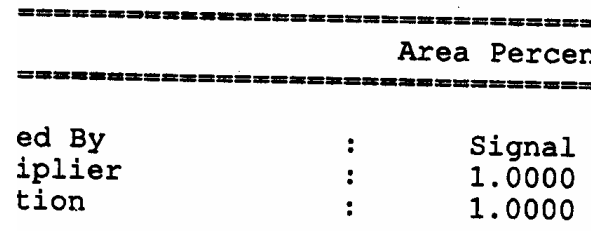

al 1: DAD1 A, Sig=250,100 $\operatorname{Ref}=360,100$

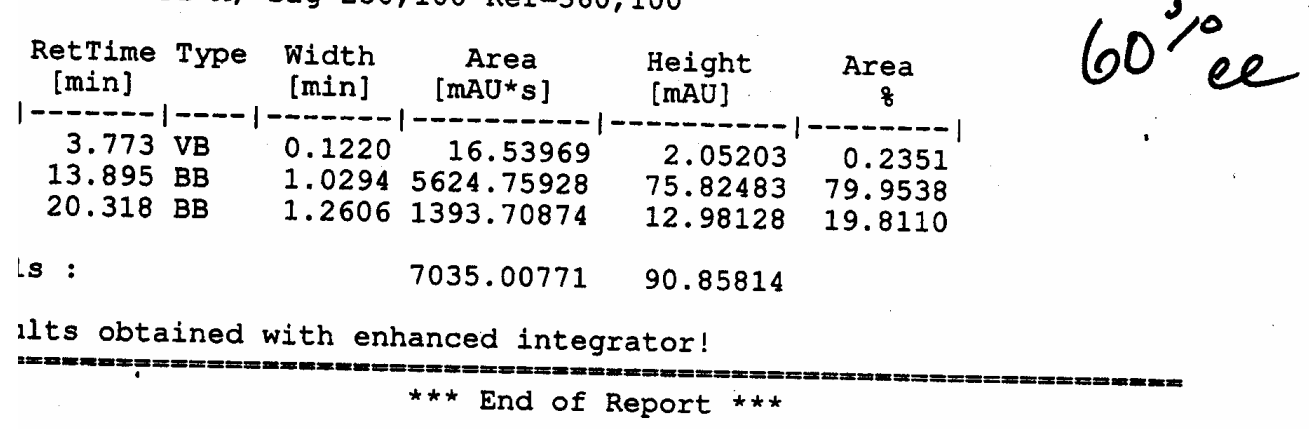

11/02 8:13:48 PM ilenden 